\title{
COMs: Complexes of Oriented Matroids
}

\author{
Hans-Jürgen Bandelt ${ }^{1}$, Victor Chepoi $^{2}$, and Kolja Knauer ${ }^{2}$ \\ ${ }^{1}$ Fachbereich Mathematik, Universität Hamburg, \\ Bundesstr. 55, 20146 Hamburg, Germany, \\ bandelt@math. uni-hamburg.de \\ ${ }^{2}$ Laboratoire d'Informatique Fondamentale, Aix-Marseille Université and CNRS, \\ Faculté des Sciences de Luminy, F-13288 Marseille Cedex 9, France \\ \{victor.chepoi, kolja.knauer\}@lif.univ-mrs.fr
}

\begin{abstract}
In his seminal 1983 paper, Jim Lawrence introduced lopsided sets and featured them as asymmetric counterparts of oriented matroids, both sharing the key property of strong elimination. Moreover, symmetry of faces holds in both structures as well as in the so-called affine oriented matroids. These two fundamental properties (formulated for covectors) together lead to the natural notion of "conditional oriented matroid" (abbreviated COM). These novel structures can be characterized in terms of three cocircuits axioms, generalizing the familiar characterization for oriented matroids. We describe a binary composition scheme by which every COM can successively be erected as a certain complex of oriented matroids, in essentially the same way as a lopsided set can be glued together from its maximal hypercube faces. A realizable COM is represented by a hyperplane arrangement restricted to an open convex set. Among these are the examples formed by linear extensions of ordered sets, generalizing the oriented matroids corresponding to the permutohedra. Relaxing realizability to local realizability, we capture a wider class of combinatorial objects: we show that non-positively curved Coxeter zonotopal complexes give rise to locally realizable COMs.
\end{abstract}

Keywords: oriented matroid, lopsided set, cell complex, tope graph, cocircuit, Coxeter zonotope.

\section{Contents}

1. Introduction 2

1.1. Avant-propos 2

1.2. Realizable COMs: motivating example 3

1.3. Properties of realizable COMs 4

1.4. Structure of the paper 6

2. Basic axioms 6

3. Minors, fibers, and faces 8

4. Tope graphs 11

5. Minimal generators of strong elimination systems 12

6. Cocircuits of COMs 16

7. Hyperplanes, carriers, and halfspaces 18

8. Decomposition and amalgamation 21

9. Euler-Poincaré formulae 22

10. Ranking COMs 24

11. COMs as complexes of oriented matroids 29

11.1. Regular cell complexes 29 
11.3. Cell complexes of COMs 30

11.4. Zonotopal COMs 31

11.5. $\mathrm{CAT}(0)$ Coxeter COMs 32

12. Concluding remarks 38

References 39

\section{INTRODUCTION}

1.1. Avant-propos. Co-invented by Bland \& Las Vergnas [11] and Folkman \& Lawrence [21], and further investigated by Edmonds \& Mandel [20] and many other authors, oriented matroids represent a unified combinatorial theory of orientations of ordinary matroids, which simultaneously captures the basic properties of sign vectors representing the regions in a hyperplane arrangement in $\mathbb{R}^{n}$ and of sign vectors of the circuits in a directed graph. Furthermore, oriented matroids find applications in point and vector configurations, convex polytopes, and linear programming. Just as ordinary matroids, oriented matroids may be defined in a multitude of distinct but equivalent ways: in terms of cocircuits, covectors, topes, duality, basis orientations, face lattices, and arrangements of pseudospheres. A full account of the theory of oriented matroids is provided in the book by Björner, Las Vergnas, White, and Ziegler 10 and an introduction to this rich theory is given in the textbook by Ziegler [39].

Lopsided sets of sign vectors defined by Lawrence $[29]$ in order to capture the intersection patterns of convex sets with the orthants of $\mathbb{R}^{d}$ (and further investigated in 3,4 ) have found numerous applications in statistics, combinatorics, learning theory, and computational geometry, see e.g. 34 for further details. Lopsided sets represent an "asymmetric offshoot" of oriented matroid theory. According to the topological representation theorem, oriented matroids can be viewed as regular CW cell complexes decomposing the $(d-1)$-sphere. Lopsided sets on the other hand can be regarded as particular contractible cubical complexes.

In this paper we propose a common generalization of oriented matroids and lopsided sets which is so natural that it is surprising that it was not discovered much earlier. It also generalizes such well-known and useful structures as convex geometries and CAT(0) cube (and zonotopal) complexes. In this generalization, global symmetry and the existence of the zero sign vector, required for oriented matroids, are replaced by local relative conditions. Analogous to conditional lattices (see [22, p. 93]) and conditional antimatroids (which are particular lopsided sets [3]), this motivates the name "conditional oriented matroids" (abbreviated: $\mathrm{COMs}$ ) for these new structures. Furthermore, COMs can be viewed as complexes whose cells are oriented matroids and which are glued together in a lopsided fashion. To illustrate the concept of a COM and compare it with similar notions of oriented matroids and lopsided sets, we continue by describing the geometric model of realizable COMs. 
1.2. Realizable COMs: motivating example. Let us begin by considering the following familiar scenario of hyperplane arrangements and realizable oriented matroids; compare with [10, Sections 2.1, 4.5] or [39, p. 212]. Given a central arrangement of hyperplanes of $\mathbb{R}^{d}$ (i.e., a finite set $E$ of $(d-1)$-dimensional linear subspaces of $\mathbb{R}^{d}$ ), the space $\mathbb{R}^{d}$ is partitioned into open regions and recursively into regions of the intersections of some of the given hyperplanes. Specifically, we may encode the location of any point from all these regions relative to this arrangement when for each hyperplane one of the corresponding halfspaces is regarded as positive and the other one as negative. Zero designates location on that hyperplane. Then the set $\mathcal{L}$ of all sign vectors representing the different regions relative to $E$ is the set of covectors of the oriented matroid of the arrangement $E$. The oriented matroids obtained in this way are called realizable. If instead of a central arrangement one considers finite arrangements $E$ of affine hyperplanes (an affine hyperplane is the translation of a (linear) hyperplane by a vector), then the sets of sign vectors of regions defined by $E$ are known as realizable affine oriented matroids [27] and [3, p.186]. Since an affine arrangement on $\mathbb{R}^{d}$ can be viewed as the intersection of a central arrangement of $\mathbb{R}^{d+1}$ with a translate of a coordinate hyperplane, each realizable affine oriented matroid can be embedded into a larger realizable oriented matroid.

Now suppose that $E$ is a central or affine arrangement of hyperplanes of $\mathbb{R}^{d}$ and $C$ is an open convex set, which may be assumed to intersect all hyperplanes of $E$ in order to avoid redundancy. Restrict the arrangement pattern to $C$, that is, remove all sign vectors which represent the open regions disjoint from $C$. Denote the resulting set of sign vectors by $\mathcal{L}(E, C)$ and call it a realizable $C O M$. Figure $1(\mathrm{a})$ displays an arrangement comprising two pairs of parallel lines and a fifth line intersecting the former four lines within the open 4-gon. Three lines (nos. 2, 3, and 5) intersect in a common point. The line arrangement defines 11 open regions within the open 4 -gon, which are represented by their topes, viz. \pm 1 covectors. The dotted lines connect adjacent topes and thus determine the tope graph of the arrangement. This graph is shown in Figure 1(b) unlabeled, but augmented by the covectors of the 14 one-dimensional and 4 two-dimensional faces.

Our model of realizable COMs generalizes realizability of oriented and affine oriented matroids on the one hand and realizability of lopsided sets on the other hand. In the case of a central arrangement $E$ with $C$ being any open convex set containing the origin (e.g., the open unit ball or the entire space $\left.\mathbb{R}^{d}\right)$, the resulting set $\mathcal{L}(E, C)$ of sign vectors coincides with the realizable oriented matroid of $E$. If the arrangement $E$ is affine and $C$ is the entire space, then $\mathcal{L}(E, C)$ coincides with the realizable affine oriented matroid of $E$. The realizable lopsided sets arise by taking the (central) arrangement $E$ of all coordinate hyperplanes $E$ restricted to arbitrary open convex sets $C$ of $\mathbb{R}^{d}$. In fact, the original definition of realizable lopsided sets by Lawrence 29 is similar but used instead an arbitrary (not necessarily open) convex set $K$ and as regions the closed orthants. Clearly, $K$ can be assumed to be a polytope, namely the convex hull of points representing the closed orthants meeting $K$. Whenever the polytope $K$ does not meet a closed orthant then some open neighborhood of $K$ does not meet that orthant either. Since there are only finitely many orthants, the intersection of 


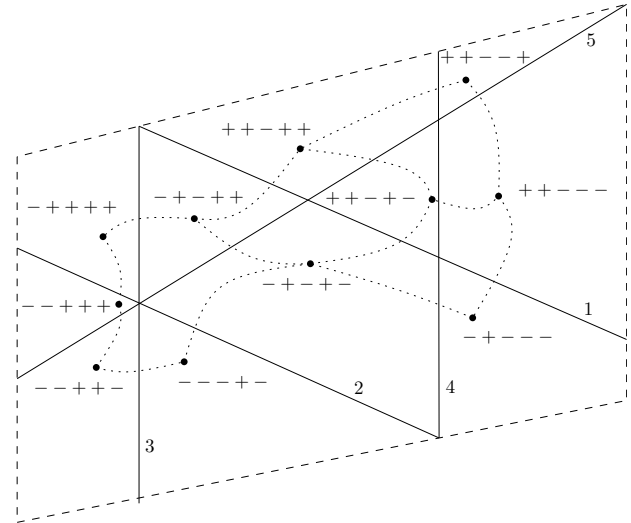

(a)

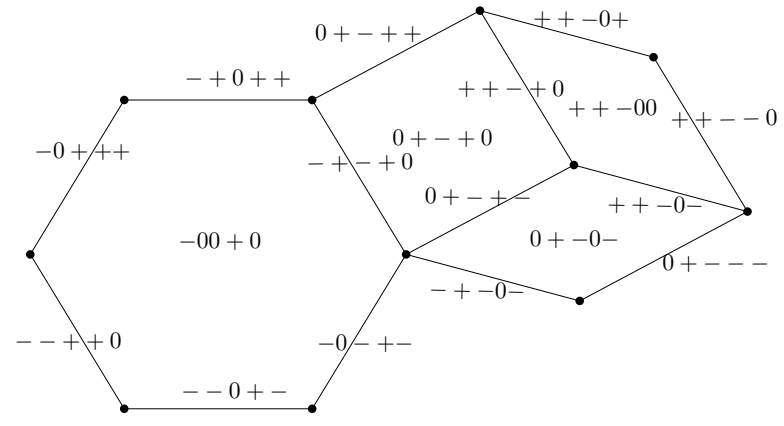

(b)

Figure 1. (a) An arrangement of five lines and its tope graph. (b) Faces and edges of the tope graph are labeled with corresponding covectors. Sign vectors are abbreviated as strings of,+- , and 0 and to be read from left to right.

these open neighborhoods results in an open set $C$ which has the same intersection pattern with the closed orthants as $K$. Now, if an open set meets a closed orthant it will also meet the corresponding open orthant, showing that both concepts of realizable lopsided sets coincide.

1.3. Properties of realizable COMs. For the general scenario of realizable COMs, we can attempt to identify its basic properties that are known to hold in oriented matroids. Let $X$ and $Y$ be sign vectors belonging to $\mathcal{L}$, thus designating regions represented by two points $x$ and $y$ within $C$ relative to the arrangement $E$; see Figure 2 (compare with Fig. 4.1 .1 of $[10]$ ). Connect the two points by a line segment and choose $\epsilon>0$ small enough so that the open ball of radius $\epsilon$ around $x$ intersects only those hyperplanes from $E$ on which $x$ lies. Pick any point $w$ from the intersection of this $\epsilon$-ball with the open line segment between $x$ and $y$. Then the corresponding sign vector $W$ is the composition $X \circ Y$ as defined by

$$
(X \circ Y)_{e}=X_{e} \text { if } X_{e} \neq 0 \text { and }(X \circ Y)_{e}=Y_{e} \text { if } X_{e}=0 .
$$

Hence the following rule is fulfilled:

(Composition) $X \circ Y$ belongs to $\mathcal{L}$ for all sign vectors $X$ and $Y$ from $\mathcal{L}$.

If we select instead a point $u$ on the ray from $y$ via $x$ within the $\epsilon$-ball but beyond $x$, then the corresponding sign vector $U$ has the opposite signs relative to $W$ at the coordinates corresponding to the hyperplanes from $E$ on which $x$ is located and which do not include the ray from $y$ via $x$. Therefore the following property holds:

(Face symmetry) $X \circ-Y$ belongs to $\mathcal{L}$ for all $X, Y$ in $\mathcal{L}$. 
Next assume that the hyperplane $e$ from $E$ separates $x$ and $y$, that is, the line segment between $x$ and $y$ crosses $e$ at some point $z$. The corresponding sign vector $Z$ is then zero at $e$ and equals the composition $X \circ Y$ at all coordinates where $X$ and $Y$ are sign-consistent, that is, do not have opposite signs:

(Strong elimination) for each pair $X, Y$ in $\mathcal{L}$ and for each $e \in E$ with $X_{e} Y_{e}=-1$ there exists $Z \in \mathcal{L}$ such that $Z_{e}=0$ and $Z_{f}=(X \circ Y)_{f}$ for all $f \in E$ with $X_{f} Y_{f} \neq-1$.

Now, the single property of oriented matroids that we have missed in the general scenario is the existence of the zero sign vector, which would correspond to a non-empty intersection of all hyperplanes from $E$ within the open convex set $C$ :

(Zero vector) the zero sign vector $\mathbf{0}$ belongs to $\mathcal{L}$.

On the other hand, if the hyperplanes from $E$ happen to be the coordinate hyperplanes, then wherever a sign vector $X$ has zero coordinates, the composition of $X$ with any sign vector from $\{ \pm 1,0\}^{E}$ is a sign vector belonging to $\mathcal{L}$. This rule, which is stronger than composition and face symmetry, holds in lopsided systems, for which the "tope" sets are exactly the lopsided sets sensu Lawrence [29]:

(Ideal composition) $X \circ Y \in \mathcal{L}$ for all $X \in \mathcal{L}$ and all sign vectors $Y$, that is, substituting any zero coordinate of a sign vector from $\mathcal{L}$ by any other sign yields a sign vector of $\mathcal{L}$.

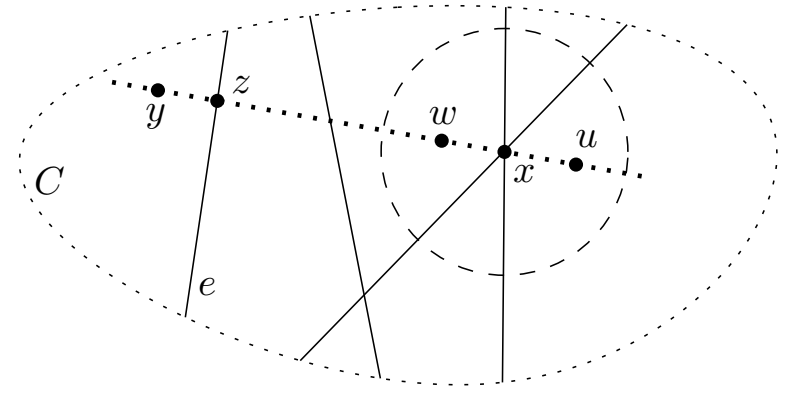

FiguRE 2. Motivating model for the three axioms.

In the model of hyperplane arrangements we can retrieve the cells which constitute oriented matroids. Indeed, consider all non-empty intersections of hyperplanes from $E$ that are minimal with respect to inclusion. Select any sufficiently small open ball around some point from each intersection. Then the subarrangement of hyperplanes through each of these points determines regions within these open balls which yield an oriented matroid of sign vectors. Taken together all these constituents form a complex of oriented matroids, where their intersections are either empty or are faces of the oriented matroids involved. These complexes are still quite special as they conform to global strong elimination. The latter feature is not guaranteed in general complexes of oriented matroids, which were called "bouquets of oriented matroids" 15 . 
It is somewhat surprising that the generalization of oriented matroids defined by the three fundamental properties of composition, face symmetry, and strong elimination have apparently not yet been studied systematically. On the other hand, the preceding discussion shows that the properties of composition and strong elimination hold whenever $C$ is an arbitrary convex set. We used the hypothesis that the set $C$ be open only for deriving face symmetry. The following example shows that indeed face symmetry may be lost when $C$ is closed: take two distinct lines in the Euclidean plane, intersecting in some point $x$ and choose as $C$ a closed halfspace which includes $x$ and the entire ++ region but is disjoint from the -region. Then $+-,+0,++, 0+,-+$, and 00 comprise the sign vectors of the regions within $C$, thus violating face symmetry. Indeed, the obtained system can be regarded as a lopsided system with an artificial zero added. On the other hand, one can see that objects obtained this way are realizable oriented matroid polyhedra [10, p. 420].

1.4. Structure of the paper. In Section 2 we will continue by formally introducing the systems of sign vectors considered in this paper. In Section 3 we prove that COMs are closed under minors and simplification, thus sharing this fundamental property with oriented matroids. We also introduce the fundamental concepts of fibers and faces of COMs, and show that faces of COMs are OMs. Section 4 is dedicated to topes and tope graphs of COMs and we show that both these objects uniquely determine a COM. Section 5 is devoted to characterizations of minimal systems of sign-vectors which generate a given COM by composition. In Section 6 we extend these characterizations and, analogously to oriented matroids, obtain a characterization of COMs in terms of cocircuits. In Section 7 we define carriers, hyperplanes, and halfspaces, all being COMs naturally contained in a given COM. We present a characterization of COMs in terms of these substructures. In Section 8 we study decomposition and amalgamation procedures for COMs and show that every COM can be obtained by successive amalgamation of oriented matroids. In Section 9, we extend the Euler-Poincaré formula from OMs to COMs and characterize lopsided sets in terms of a particular variant of it. In Section 10 as a resuming example we study the COMs provided by the ranking extensions - aka weak extensions - of a partially ordered set and illustrate the operations and the results of the paper on them. In Section 11 we consider a topological approach to COMs and study them as complexes of oriented matroids. In particular, we show that non-positively curved Coxeter zonotopal complexes give rise to COMs. We close the paper with several concluding remarks and two conjectures in Section 12 .

\section{BASIC AXIOMS}

We follow the standard oriented matroids notation from [10]. Let $E$ be a non-empty finite (ground) set and let $\mathcal{L}$ be a non-empty set of sign vectors, i.e., maps from $E$ to $\{ \pm 1,0\}=$ $\{-1,0,+1\}$. The elements of $\mathcal{L}$ are also referred to as covectors and denoted by capital letters $X, Y, Z$, etc. For $X \in \mathcal{L}$, the subset $\underline{X}=\left\{e \in E: X_{e} \neq 0\right\}$ is called the support of $X$ and its complement $X^{0}=E \backslash \underline{X}=\left\{e \in E: X_{e}=0\right\}$ the zero set of $X$ (alias the kernel of $X$ ). Simlaly, we denote $X^{+}=\left\{e \in E: X_{e}=+\right\}$ and $X^{-}=\left\{e \in E: X_{e}=-\right\}$. We can regard a 
sign vector $X$ as the incidence vector of a \pm 1 signed subset $\underline{X}$ of $E$ such that to each element of $E$ one element of the signs $\{ \pm 1,0\}$ is assigned. We denote by $\leq$ the product ordering on $\{ \pm 1,0\}^{E}$ relative to the standard ordering of signs with $0 \leq-1$ (sic!) and $0 \leq+1$.

For $X, Y \in \mathcal{L}$, we call $S(X, Y)=\left\{f \in E: X_{f} Y_{f}=-1\right\}$ the separator of $X$ and $Y$. The elements of $S(X, Y)$ are said to separate $X$ and $Y$. If the separator is empty, then $X$ and $Y$ are said to be sign-consistent. In particular, this is the case when $X$ is below $Y$, that is, $X \leq Y$ holds. The composition of $X$ and $Y$ is the sign vector $X \circ Y$, where $(X \circ Y)_{e}=X_{e}$ if $X_{e} \neq 0$ and $(X \circ Y)_{e}=Y_{e}$ if $X_{e}=0$. Note that $X \leq X \circ Y$ for all sign vectors $X, Y$.

Given a set of sign vectors $\mathcal{L}$, its topes are the maximal elements of $\mathcal{L}$ with respect to $\leq$. Further let

$$
\uparrow \mathcal{L}:=\left\{Y \in\{ \pm 1,0\}^{E}: X \leq Y \text { for some } X \in \mathcal{L}\right\}=\left\{X \circ W: X \in \mathcal{L} \text { and } W \in\{ \pm 1,0\}^{E}\right\}
$$

be the upset of $\mathcal{L}$ in the ordered set $\left(\{ \pm 1,0\}^{E}, \leq\right)$.

If a set of sign vectors is closed with respect to $\circ$, then the resulting idempotent semigroup (indeed a left regular band alias skew semilattice [31, 32]) is called the braid semigroup, see e.g. [8]. The composition operation naturally occurs also elsewhere: for a single coordinate, the composite $x \circ y$ on $\{ \pm 1,0\}$ is actually derived as the term $t(x, 0, y)$ (using 0 as a constant) from the ternary discriminator $t$ on $\{ \pm 1,0\}$, which is defined by $t(a, b, c)=a$ if $a \neq b$ and $t(a, b, c)=c$ otherwise. Then in this context of algebra and logic, "composition" on the product $\{ \pm 1,0\}^{E}$ would rather be referred to as a "skew Boolean join" [6].

We continue with the formal definition of the main axioms as motivated and discussed in the previous section.

\section{Composition:}

(C) $X \circ Y \in \mathcal{L}$ for all $X, Y \in \mathcal{L}$.

Condition (C) is taken from the list of axioms for oriented matroids. Since $\circ$ is associative, arbitrary finite compositions can be written without bracketing $X_{1} \circ \ldots \circ X_{k}$ so that (C) entails that they all belong to $\mathcal{L}$. Note that contrary to a convention sometimes made in oriented matroids we do not consider compositions over an empty index set, since this would imply that the zero sign vector belonged to $\mathcal{L}$. We highlight condition $(\mathrm{C})$ here although it will turn out to be a consequence of another axiom specific in this context. The reason is that we will later use several weaker forms of the axioms which are no longer consequences from one another.

\section{Strong elimination:}

(SE) for each pair $X, Y \in \mathcal{L}$ and for each $e \in S(X, Y)$ there exists $Z \in \mathcal{L}$ such that $Z_{e}=0$ and $Z_{f}=(X \circ Y)_{f}$ for all $f \in E \backslash S(X, Y)$.

Note that $(X \circ Y)_{f}=(Y \circ X)_{f}$ holds exactly when $f \in E \backslash S(X, Y)$. Therefore the sign vector $Z$ provided by (SE) serves both ordered pairs $X, Y$ and $Y, X$.

Condition (SE) is one of the axioms for covectors of oriented matroids and is implied by the property of route systems in lopsided sets, see Theorem 5 of [29]. 


\section{Symmetry:}

(Sym) $-\mathcal{L}=\{-X: X \in \mathcal{L}\}=\mathcal{L}$, that is, $\mathcal{L}$ is closed under sign reversal.

Symmetry restricted to zero sets of covectors (where corresponding supports are retained) is dubbed:

\section{Face symmetry:}

(FS) $X \circ-Y \in \mathcal{L}$ for all $X, Y \in \mathcal{L}$.

This condition can also be expressed by requiring that for each pair $X, Y$ in $\mathcal{L}$ there exists $Z \in \mathcal{L}$ with $X \circ Z=Z$ such that $X=\frac{1}{2}(X \circ Y+X \circ Z)$. Face symmetry trivially implies (C) because by (FS) we first get $X \circ-Y \in \mathcal{L}$ and then $X \circ Y=(X \circ-X) \circ Y=X \circ-(X \circ-Y)$ for all $X, Y \in \mathcal{L}$.

\section{Ideal composition:}

(IC) $\uparrow \mathcal{L}=\mathcal{L}$.

Notice that (IC) implies (C) and (FS). We are now ready to define the main objects of our study:

Definition 1. A system of sign vectors $(E, \mathcal{L})$ is called a:

- strong elimination system if $\mathcal{L}$ satisfies $(\mathrm{C})$ and $(\mathrm{SE})$,

- conditional oriented matroid (COM) if $\mathcal{L}$ satisfies $(\mathrm{FS})$ and $(\mathrm{SE})$,

- oriented matroid (OM) if $\mathcal{L}$ satisfies $(\mathrm{C}),(\mathrm{Sym})$, and $(\mathrm{SE})$,

- lopsided system if $\mathcal{L}$ satisfies (IC) and (SE).

For oriented matroids one can replace (C) and (Sym) by (FS) and

\section{Zero vector:}

(Z) the zero sign vector $\mathbf{0}$ belongs to $\mathcal{L}$.

Notice that the axiom (SE) can be somewhat weakened in the presence of (C), i.e., in particular in Definition 1. If $(\mathrm{C})$ is true in the system $(E, \mathcal{L})$, then for $X, Y \in \mathcal{L}$ we have $X \circ Y=(X \circ Y) \circ(Y \circ X), \underline{X \circ Y}=\underline{Y \circ X}=\underline{X} \cup \underline{Y}$, and also for the separators we have $S(X \circ Y, Y \circ X)=S(X, Y)$.

Therefore, if (C) holds, we may substitute (SE) by

$\left(\mathbf{S E}^{=}\right)$for each pair $X, Y \in \mathcal{L}$ with $\underline{X}=\underline{Y}$ and for each $e \in S(X, Y)$ there exists $Z \in \mathcal{L}$ such that $Z_{e}=0$ and $Z_{f}=(X \circ Y)_{f}$ for all $f \in E \backslash S(X, Y)$,

The axioms (C), (FS), ( $\mathrm{SE}^{=}$) (plus a fourth condition) were used by Karlander [27] in his study of affine oriented matroids that are embedded as "halfspaces" (see Section 7 below) of oriented matroids.

\section{Minors, FiBERS, AND FACES}

In the present technical section we show that the class of COMs is closed under taking minors, defined as for oriented matroids. We use this to establish that simplifications and 
semisimplifications of COMs are minors of COMs and therefore COMs. We also introduce fibers and faces of COMs, which will be of importance for the rest of the paper.

Let $(E, \mathcal{L})$ be a COM and $A \subseteq E$. Given a sign vector $X \in\{ \pm 1,0\}^{E}$ by $X \backslash A$ we refer to the restriction of $X$ to $E \backslash A$, that is $X \backslash A \in\{ \pm 1,0\}^{E \backslash A}$ with $(X \backslash A)_{e}=X_{e}$ for all $e \in E \backslash A$. The deletion of $A$ is defined as $(E \backslash A, \mathcal{L} \backslash A)$, where $\mathcal{L} \backslash A:=\{X \backslash A: X \in \mathcal{L}\}$. The contraction of $A$ is defined as $(E \backslash A, \mathcal{L} / A)$, where $\mathcal{L} / A:=\{X \backslash A: X \in \mathcal{L}$ and $\underline{X} \cap A=\varnothing\}$. If a system of sign vectors arises by deletions and contractions from another one it is said to be minor of it.

Lemma 1. The properties $(\mathrm{C}),(\mathrm{FS})$, and (SE) are all closed under taking minors. In particular, if $(E, \mathcal{L})$ is a $C O M$ and $A \subseteq E$, then $(E \backslash A, \mathcal{L} \backslash A)$ and $(E \backslash A, \mathcal{L} / A)$ are COMs as well.

Proof. We first prove that $(E \backslash A, \mathcal{L} \backslash A)$ is a COM. To see (C) and (FS) let $X \backslash A, Y \backslash A \in \mathcal{L} \backslash A$. Then $X \circ( \pm Y) \in \mathcal{L}$ and $(X \circ( \pm Y)) \backslash A=X \backslash A \circ( \pm Y \backslash A) \in \mathcal{L} \backslash A$.

To see (SE) let $X \backslash A, Y \backslash A \in \mathcal{L} \backslash A$ and $e$ an element separating $X \backslash A$ and $Y \backslash A$. Then there is $Z \in \mathcal{L}$ with $Z_{e}=0$ and $Z_{f}=(X \circ Y)_{f}$ for all $f \in E \backslash S(X, Y)$. Clearly, $Z \backslash A \in \mathcal{L} \backslash A$ satisfies (SE) with respect to $X \backslash A, Y \backslash A$.

Now, we prove that $(E \backslash A, \mathcal{L} / A)$ is a COM. Let $X \backslash A, Y \backslash A \in \mathcal{L} / A$, i.e., $\underline{X} \cap A=\underline{Y} \cap A=\varnothing$. Hence $X \circ( \pm Y) \cap A=\varnothing$ and therefore $X \backslash A \circ( \pm Y \backslash A) \in \mathcal{L} / A$, proving (C) and (FS).

To see (SE) let $X \backslash A, Y \backslash A \in \mathcal{L} / A$ and $e$ an element separating $X \backslash A$ and $Y \backslash A$. Then there is $Z \in \mathcal{L}$ with $Z_{e}=0$ and $Z_{f}=(X \circ Y)_{f}$ for all $f \in E \backslash S(X, Y)$. In particular, if $X_{f}=Y_{f}=0$, then $Z_{f}=0$. Therefore, $Z \backslash A \in \mathcal{L} / A$ and it satisfies (SE).

Lemma 2. If $(E, \mathcal{L})$ is a system of sign vectors and $A, B \subseteq E$ with $A \cap B=\varnothing$, then $(E \backslash(A \cup B),(\mathcal{L} \backslash A) / B)=(E \backslash(A \cup B),(\mathcal{L} / B) \backslash A)$.

Proof. It suffices to prove this for $A$ and $B$ consisting of single elements $e$ and $f$, respectively. Now $X \backslash\{e, f\} \in(\mathcal{L} \backslash\{e\}) /\{f\}$ if and only if $X \in \mathcal{L}$ with $X_{f}=0$ which is equivalent to $X \backslash\{e\} \in \mathcal{L} \backslash\{e\}$ with $(X \backslash\{e\})_{f}=0$. This is, $X \backslash\{e, f\} \in(\mathcal{L} /\{f\}) \backslash\{e\}$.

Next, we will define simple and semisimple systems of sign vectors. These are motivated by the hyperplane model for COMs, that possesses additional properties, reflecting that we have a set of hyperplanes rather than a multiset and that the given convex set is open. This is also motivated by the requirement of defining systems of sign vectors not containing coloops and parallel elements, which is relevant, for example, for the identifications of topes.

A coloop of $(E, \mathcal{L})$ is an element $e \in E$ such that $X_{e}=0$ for all $X \in \mathcal{L}$. Hence $(E, \mathcal{L})$ does not have coloops if and only if for each element $e$, there exists a covector $X$ with $X_{e} \neq 0$. Two elements $e, e^{\prime} \in E$ of $(E, \mathcal{L})$ are parallel, denoted $e \| e^{\prime}$, if either $X_{e}=X_{e^{\prime}}$ for all $X \in \mathcal{L}$ or $X_{e}=-X_{e^{\prime}}$ for all $X \in \mathcal{L}$. It is easy to see that $\|$ is an equivalence relation. The condition that $(E, \mathcal{L})$ does not contain parallel elements can be expressed by the requirement that for each pair $e \neq f$ in $E$, there exist $X, Y \in \mathcal{L}$ with $X_{e} \neq X_{f}$ and $Y_{e} \neq-Y_{f}$. 
Simple systems are defined by two axioms which are slightly stronger than those which ensure that the absence of coloops and parallel elements. We call the system $(E, \mathcal{L})$ simple if it satisfies the following non-redundancy axioms:

\section{Simplicity:}

(N1) for each $e \in E,\{ \pm 1,0\}=\left\{X_{e}: X \in \mathcal{L}\right\}$;

(N2) for each pair $e \neq f$ in $E$, there exist $X, Y \in \mathcal{L}$ with $\left\{X_{e} X_{f}, Y_{e} Y_{f}\right\}=\{ \pm 1\}$.

We will also consider the weaker notion of semisimple systems, which are the simple systems when restricted to the set

$$
E_{ \pm}:=\left\{e \in E:\left\{X_{e}: X \in \mathcal{L}\right\} \neq\{+1\},\{-1\}\right\}
$$

of those elements $e$ at which $\mathcal{L}$ is not non-zero constant. We call the system $(E, \mathcal{L})$ semisimple if it satisfies the following restricted non-redundancy axioms:

\section{Semisimplicity:}

(RN1) for each $e \in E_{ \pm},\{ \pm 1,0\}=\left\{X_{e}: X \in \mathcal{L}\right\}$

(RN2) for each pair $e \neq f$ in $E_{ \pm}$, there exist $X, Y \in \mathcal{L}$ with $\left\{X_{e} X_{f}, Y_{e} Y_{f}\right\}=\{ \pm 1\}$.

Let $E_{0}:=\left\{e \in E: X_{e}=0\right.$ for all $\left.X \in \mathcal{L}\right\}$ be the set of all coloops. Condition (RN1) yields that $E_{0}=\varnothing$. The condition that there are no coloops is relevant for the identification of topes. Recall that a tope of $\mathcal{L}$ is any covector $X$ that is maximal with respect to the standard sign ordering defined above. In the presence of $(\mathrm{C})$, the covector $X$ is a tope precisely when $X \circ Y=X$ for all $Y \in \mathcal{L}$, that is, for each $e \in E$ either $X_{e} \in\{ \pm 1\}$ or $Y_{e}=0$ for all $Y \in \mathcal{L}$. In particular, if both $(\mathrm{C})$ and $E_{0}=\varnothing$ hold, then the topes are exactly the covectors with full support $E$. Notice also that condition (N2) yields that there are no parallel elements. Consequently, simple systems do not contain coloops, parallel elements, and their topes are the covectors with full support (while semisimple systems satisfy the first and the third conditions).

Further put

$$
\begin{gathered}
E_{1}:=\left\{e \in E: \#\left\{X_{e}: X \in \mathcal{L}\right\}=1\right\}=E_{0} \cup\left(E \backslash E_{ \pm}\right), \\
E_{2}:=\left\{e \in E: \#\left\{X_{e}: X \in \mathcal{L}\right\}=2\right\} .
\end{gathered}
$$

The sets $E_{0} \cup E_{2}$ and $E_{1} \cup E_{2}$ comprise the positions at which $\mathcal{L}$ violates $(\mathrm{RN} 1)$ or $(\mathrm{N} 1)$, respectively. Hence the deletions $\left(E \backslash\left(E_{0} \cup E_{2}\right), \mathcal{L} \backslash\left(E_{0} \cup E_{2}\right)\right)$ and $\left(E \backslash\left(E_{1} \cup E_{2}\right), \mathcal{L} \backslash\left(E_{1} \cup E_{2}\right)\right)$ constitute the canonical transforms of $(E, \mathcal{L})$ satisfying $(\mathrm{RN} 1)$ and $(\mathrm{N} 1)$, respectively. One equivalence class of the parallel relation $\|$ restricted to $\mathcal{L} \backslash\left(E_{0} \cup E_{2}\right)$ is $E \backslash E_{ \pm}$, i.e., it comprises the (non-zero) constant positions. Exactly this class gets removed when one restricts $\|$ further to $\mathcal{L} \backslash\left(E_{1} \cup E_{2}\right)$. Selecting a transversal for the classes of $\|$ on $\mathcal{L} \backslash\left(E_{1} \cup E_{2}\right)$, which arbitrarily picks exactly one element from each class and deletes all others, results in a simple system. Restoring the entire class $E \backslash E_{ \pm}$then yields a semisimple system. We refer to these canonical transforms as to the simplification and semisimplification of $(E, \mathcal{L})$, respectively. Then from Lemma 1 we obtain: 
Lemma 3. The semisimplification of a system $(E, \mathcal{L})$ of sign vectors is a semisimple minor and the simplification is a simple minor, unique up to sign reversal on subsets of $E_{ \pm}$. Either system is a $C O M$ whenever $(E, \mathcal{L})$ is.

For a system $(E, \mathcal{L})$, a fiber relative to some $X \in \mathcal{L}$ and $A \subseteq E$ is a set of sign vectors defined by

$$
\mathcal{R}=\{Y \in \mathcal{L}: Y \backslash A=X \backslash A\} .
$$

We say that such a fiber is topal if $(X \backslash A)^{0}=\varnothing$, that is, $X \backslash A$ is a tope of the minor $(E \backslash A, \mathcal{L} \backslash A) . \mathcal{R}$ is a face if $X$ can be chosen so that $X^{0}=A$, whence faces are topal fibers. Note that the entire system $(E, \mathcal{L})$ can be regarded both as a topal fiber and as the result of an empty deletion or contraction. If $(E, \mathcal{L})$ satisfies $(\mathrm{C})$, then the fiber relative to $A:=X^{0}$, alias $X$-face, associated with a sign vector $X$ can be expressed in the form

$$
F(X):=\{X \circ Y: Y \in \mathcal{L}\}=\mathcal{L} \cap \uparrow\{X\} .
$$

If $S(V, W)$ is non-empty for $V, W \in \mathcal{L}$, then the corresponding faces $F(V)$ and $F(W)$ are disjoint. Else, if $V$ and $W$ are sign-consistent, then $F(V) \cap F(W)=F(V \circ W)$. In particular $F(V) \subseteq F(W)$ is equivalent to $V \in F(W)$, that is, $W \leq V$. The ordering of faces by inclusion thus reverses the sign ordering. The following observations are straightforward and recorded here for later use:

Lemma 4. If $(E, \mathcal{L})$ is a strong elimination system or a $C O M$, respectively, then so are all fibers of $(E, \mathcal{L})$. If $(E, \mathcal{L})$ is semisimple, then so is every topal fiber. If $(E, \mathcal{L})$ is a COM, then for any $X \in \mathcal{L}$ the minor $(E \backslash \underline{X}, F(X) \backslash \underline{X})$ corresponding to the face $F(X)$ is an OM, which is simple whenever $(E, \mathcal{L})$ is semisimple.

\section{TOPE GRAPHS}

One may wonder whether and how the topes of a semisimple COM $(E, \mathcal{L})$ determine and generate $\mathcal{L}$. We cannot avoid using face symmetry because one can turn every COM which is not an $\mathrm{OM}$ into a strong elimination system by adding the zero vector to the system, without affecting the topes. The following result for simple oriented matroids was first observed by Mandel (unpublished), see [10, Theorem 4.2.13].

Proposition 1. Every semisimple $\operatorname{COM}(E, \mathcal{L})$ is uniquely determined by its set of topes.

Proof. We proceed by induction on \#E. For a single position the assertion is trivial. So assume $\# E \geq 2$. Let $\mathcal{L}$ and $\mathcal{L}^{\prime}$ be two COMs on $E$ sharing the same set of topes. Then deletion of any $g \in E$ results in two COMs with equal tope sets, whence $\mathcal{L}^{\prime} \backslash g=\mathcal{L} \backslash g$ by the induction hypothesis. Suppose that there exists some $W \in \mathcal{L}^{\prime} \backslash \mathcal{L}$ chosen with $W^{0}$ as small as possible. Then $\# W^{0}>0$. Take any $e \in W^{0}$. Then as $\mathcal{L}^{\prime} \backslash e=\mathcal{L} \backslash e$ by semisimplicity there exists a sign vector $V$ in $\mathcal{L}$ such that $V \backslash e=W \backslash e$ and $V_{e} \neq 0$. Since $V^{0} \subset W^{0}$, we infer that $V \in \mathcal{L}^{\prime}$ by the minimality choice of $W$. Then, by (FS) applied to $W$ and $V$ in $\mathcal{L}^{\prime}$, we get $W \circ-V \in \mathcal{L}^{\prime}$. This sign vector also belongs to $\mathcal{L}$ because $\#(W \circ-V)^{0}=\# V^{0}<\# W^{0}$. Finally, apply (SE) to the pair $V, W \circ-V$ in $\mathcal{L}$ relative to $e$ and obtain $Z=W \in \mathcal{L}$, in conflict with the initial assumption. 
The tope graph of a semisimple COM on $E$ is the graph with all topes as its vertices where two topes are adjacent exactly when they differ in exactly one coordinate. In other words, the tope graph is the subgraph of the \#E-dimensional hypercube with vertex set $\{ \pm 1\}^{E}$ induced by the tope set. Isometry means that the internal distance in the subgraph is the same as in the hypercube. Isometric subgraphs of the hypercube are often referred to as a partial cubes [25]. For tope graphs of oriented matroids the next result was first proved in 28]

Proposition 2. The tope graph of a semisimple strong elimination system $(E, \mathcal{L})$ is a partial cube in which the edges correspond to the sign vectors of $\mathcal{L}$ with singleton zero sets.

Proof. If $X$ and $Y$ are two adjacent topes, say, differing at position $e \in E$, then the vector $Z \in \mathcal{L}$ provided by (SE) for this pair relative to $e$ has 0 at $e$ and coincides with $X$ and $Y$ at all other positions. By way of contradiction assume that now $X$ and $Y$ are two topes which cannot be connected by a path in the tope graph of length $\# S(X, Y)=k>1$ such that $k$ is as small as possible. Then the interval $[X, Y]$ consisting of all topes on shortest paths between $X$ and $Y$ in the tope graph comprises only $X$ and $Y$. For $e \in S(X, Y)$ we find some $Z \in \mathcal{L}$ such that $Z_{e}=0$ and $Z_{g}=X_{g}$ for all $g \in E \backslash S(X, Y)$ by (SE). If there exists $f \in S(X, Y) \backslash\{e\}$ with $Z_{f} \neq 0$, then $Z \circ X$ or $Z \circ Y$ is a tope different from $X$ and $Y$, but contained in $[X, Y]$, a contradiction.

If $Z_{f}=0$ for all $f \in S(X, Y) \backslash\{e\}$, then by (RN2) there is $W \in \mathcal{L}$ with $0 \neq W_{e} W_{f} \neq$ $X_{e} X_{f} \neq 0$. We conclude that $Z \circ W \circ Y$ is a tope different from $X$ and $Y$ but contained in $[X, Y]$, a contradiction. This concludes the proof.

Isometric embeddings of partial cubes into hypercubes are unique up to automorphisms of the hosting hypercube [16, Proposition 19.1.2] (and addition of superfluous dimensions). Hence, Propositions 1 and 2 together imply the following result, which generalizes a similar result of [9] for tope graphs of OMs:

Proposition 3. A simple COM is determined by its tope graph up to reorientation.

\section{Minimal generators of Strong Elimination Systems}

We have seen in the preceding section that a COM is determined by its tope set. There is a more straightforward way to generate any strong elimination system from bottom to top by taking suprema. This generation process involves only some weaker forms of the axioms $(\mathrm{C})$ and $(\mathrm{SE})$.

Let $(E, \mathcal{L})$ be a system of sign vectors. Given $X, Y \in \mathcal{L}$ consider the following set of sign vectors which partially "conform" to $X$ relative to subsets $A \subseteq S(X, Y)$ :

$$
\begin{aligned}
\mathcal{W}_{A}(X, Y) & =\left\{Z \in \mathcal{L}: Z^{+} \subseteq X^{+} \cup Y^{+}, Z^{-} \subseteq X^{-} \cup Y^{-}, \text {and } S(X, Z) \subseteq E \backslash A\right\} \\
& =\left\{Z \in \mathcal{L}: Z_{g} \in\left\{0, X_{g}, Y_{g}\right\} \text { for all } g \in E, \text { and } Z_{h} \in\left\{0, X_{h}\right\} \text { for all } h \in A\right\} .
\end{aligned}
$$

For $A=\varnothing$ we use the short-hand $\mathcal{W}(X, Y)$, i.e.,

$$
\mathcal{W}(X, Y)=\left\{Z \in \mathcal{L}: Z^{+} \subseteq X^{+} \cup Y^{+}, Z^{-} \subseteq X^{-} \cup Y^{-}\right\}
$$


and for the maximum choice $A \supseteq S(X, Y)$ we write $\mathcal{W}_{\infty}(X, Y)$, i.e.,

$$
\mathcal{W}_{\infty}(X, Y)=\{Z \in \mathcal{W}(X, Y): S(X, Z)=\varnothing\}
$$

Trivially, $X, X \circ Y \in \mathcal{W}_{A}(X, Y)$ and $\mathcal{W}_{B}(X, Y) \subseteq \mathcal{W}_{A}(X, Y)$ for $A \subseteq B \subseteq E$. Note that $S(X, Z) \subseteq S(X, Y)$ for all $Z \in \mathcal{W}(X, Y)$. Each set $\mathcal{W}_{A}(X, Y)$ is closed under composition (and trivially is a downset with respect to the sign ordering). For, if $V, W \in \mathcal{W}_{A}(X, Y)$, then $(V \circ W)^{+} \subseteq V^{+} \cup W^{+}$and $(V \circ W)^{-} \subseteq V^{-} \cup W^{-}$holds trivially, and further, if $e \in S(X, V \circ W)$, say, $e \in X^{+}$and $e \in(V \circ W)^{-} \subseteq V^{-} \cup W^{-}$, then $e \in S(X, V)$ or $e \in S(X, W)$, that is,

$$
S(X, V \circ W) \subseteq S(X, V) \cup S(X, W) \subseteq E \backslash A .
$$

Since each of the sets $\mathcal{W}_{A}(X, Y)$ is closed under composition, we may take the composition of all sign vectors in $\mathcal{W}_{A}(X, Y)$. The result may depend on the order of the constituents.

Some features of strong elimination are captured by weak elimination:

(WE) for each pair $X, Y \in \mathcal{L}$ and $e \in S(X, Y)$ there exists $Z \in \mathcal{W}(X, Y)$ with $Z_{e}=0$.

Condition (WE) is in general weaker than (SE): consider, e.g., the four sign vectors ,,,+++--- 00 ; the zero vector $Z$ would serve all pairs $X, Y$ for (WE) but for $X=++$ and $Y=+-(\mathrm{SE})$ would require the existence of +0 rather than 00 . In the presence of (IC), the strong and the weak versions of elimination are equivalent, that is, lopsided systems are characterized by (IC) and (WE) [4. With systems satisfying (WE) one can generate lopsided systems by taking the upper sets:

Proposition $4([4])$. If $(E, \mathcal{K})$ is a system of sign vectors which satisfies $(\mathrm{WE})$, then $(E, \uparrow \mathcal{K})$ is a lopsided system.

Proof. We have to show that (WE) holds for $(E, \uparrow \mathcal{K})$. For $X, Y \in \uparrow \mathcal{K}$ and some element $e$ in $S(X, Y)$, pick $V, W \in \mathcal{K}$ with $V \leq X$ and $W \leq Y$. If $e \in S(V, W)$, then by (WE) in $\mathcal{K}$ one obtains some $U \in \uparrow \mathcal{K}$ such that $U_{e}=0$ and $U_{f} \leq V_{f} \circ W_{f} \leq X_{f} \circ Y_{f}$ for all $f \in E \backslash S(X, Y)$. Then the sign vector $Z$ defined by $Z_{g}:=U_{g}$ for all $g \in S(X, Y)$ and $Z_{f}:=X_{f} \circ Y_{f}$ for all $f \in E \backslash S(X, Y)$ satisfies $U \leq Z$ and hence belongs to $\uparrow \mathcal{K}$. If $e \notin S(V, W)$, then $V_{e}=0$, say. Define a sign vector $Z$ similarly as above: $Z_{g}:=V_{g}$ for $g \in S(X, Y)$ and $Z_{f}:=X_{f} \circ Y_{f} \geq V_{f}$ for $f \in E \backslash S(X, Y)$. Then $Z \in \uparrow \mathcal{K}$ is as required.

This proposition applied to a $\operatorname{COM}(E, \mathcal{L})$ yields an associated lopsided systems $(E, \uparrow \mathcal{L})$ having the same minimal sign vectors as $(E, \mathcal{L})$. This system is referred to as the lopsided envelope of $(E, \mathcal{L})$. In contrast to $(\mathrm{SE})$ and $\left(\mathrm{SE}^{=}\right)$, the following variant of strong elimination allows us to treat the positions $f \in E \backslash S(X, Y)$ one at a time:

(SE1) for each pair $X, Y \in \mathcal{L}$ and $e \in S(X, Y)$ and $f \in E \backslash S(X, Y)$ there exists $Z \in \mathcal{W}(X, Y)$ such that $Z_{e}=0$, and $Z_{f}=(X \circ Y)_{f}$.

Nevertheless, under composition axiom (C), all these variants of (SE) are equivalent:

Lemma 5. Let $(E, \mathcal{L})$ be a system of sign vectors which satisfies $(\mathrm{C})$. Then all three variants $(\mathrm{SE}),\left(\mathrm{SE}^{=}\right)$, and $(\mathrm{SE} 1)$ are equivalent. 
Proof. The equivalence of $(\mathrm{SE})$ and $\left(\mathrm{SE}^{=}\right)$in the presence of $(\mathrm{C})$ was argued at the end of Section 2. Trivially, (SE) implies (SE1). Conversely, if (SE1) holds, then for every $e \in$ $S(X, Y)$ we obtain a set $\left\{Z^{(\{e\}, f)}: f \in E \backslash S(X, Y)\right\}$ of solutions, one for each $f$. Then the composition in any order of these solutions yields a solution $Z$ for (SE), because $Z^{(\{e\}, f)} \leq Z$ for all $f \in E \backslash S(X, Y)$ and $Z_{e}=0$, whence $Z_{f}=(X \circ Y)_{f}$ for all $f \in E \backslash S(X, Y)$ and $Z_{e}=0$.

Since strong elimination captures some features of composition, one may wonder whether (C) can be somewhat weakened in the presence of (SE) or (SE1). Here suprema alias conformal compositions come into play:

(CC) $X \circ Y \in \mathcal{L}$ for all $X, Y \in \mathcal{L}$ with $S(X, Y)=\varnothing$.

Recall that $X$ and $Y$ are sign-consistent, that is, $S(X, Y)=\varnothing$ exactly when $X$ and $Y$ commute: $X \circ Y=Y \circ X$. We say that a composition $X^{(1)} \circ \ldots \circ X^{(n)}$ of sign vectors is conformal if it constitutes the supremum of $X^{(1)}, \ldots, X^{(n)}$ with respect to the sign ordering. Thus, $X^{(1)}, \ldots, X^{(n)}$ commute exactly when they are bounded from above by some sign vector, which is the case when the set of all $X_{e}^{(i)}(1 \leq i \leq n)$ includes at most one non-zero sign (where $e$ is any fixed element of $E$ ). If we wish to highlight this property we denote the supremum of $X^{(1)}, \ldots, X^{(n)}$ by $\bigodot_{i=1}^{n} X^{(i)}$ or $X^{(1)} \odot \ldots \odot X^{(n)}\left(\operatorname{instead}\right.$ of $\left.X^{(1)} \circ \ldots \circ X^{(n)}\right)$. Clearly the conformal property is Helly-type in the sense that a set of sign vectors has a supremum if each pair in that set does.

Given any system $\mathcal{K}$ of sign vectors on $E$ define $\odot \mathcal{K}$ as the set of all (non-empty) suprema of members from $\mathcal{K}$. We say that a system $(E, \mathcal{K})$ of sign vectors generates a system $(E, \mathcal{L})$ if $\odot \mathcal{K}=\mathcal{L}$. We call a sign vector $X \in \mathcal{L}$ (supremum-)irreducible if it does not equal the (non-empty!) conformal composition of any sign vectors from $\mathcal{L}$ different from $X$. Clearly, the irreducible sign vectors of $\mathcal{L}$ are unavoidable when generating $\mathcal{L}$. We denote the set of irreducibles of $\mathcal{L}$ by $\mathcal{J}=\mathcal{J}(\mathcal{L})$.

Theorem 1. Let $(E, \mathcal{L})$ be a system of sign vectors. Then the following conditions are equivalent:

(i) $(E, \mathcal{L})$ is a strong elimination system;

(ii) $\mathcal{L}$ satisfies $(\mathrm{CC})$ and (SE1);

(iii) $\mathcal{L}$ satisfies $(\mathrm{CC})$ and some set $\mathcal{K}$ with $\mathcal{J} \subseteq \mathcal{K} \subseteq \mathcal{L}$ satisfies (SE1).

(iv) $\mathcal{L}$ satisfies $(\mathrm{CC})$ and its set $\mathcal{J}$ of irreducibles satisfies (SE1).

Proof. The implication (i) $\Longrightarrow$ (ii) is trivial. Now, to see (ii) $\Longrightarrow$ (iv) let $(E, \mathcal{L})$ satisfy $(\mathrm{CC})$ and (SE1). For $X, Y \in \mathcal{J}, e \in S(X, Y)$, and $f \in E \backslash S(X, Y)$ we first obtain $Z \in \mathcal{L}$ with $Z_{e}=0$ and $Z_{f}=(X \circ Y)_{f}$. Since $Z$ is the supremum of some $Z^{(1)}, \ldots, Z^{(n)}$ from $\mathcal{J}$, there must be an index $i$ for which $Z_{f}^{(i)}=Z_{f}$ and trivially $Z_{e}^{(i)}=0$ holds. Therefore $\mathcal{J}$ satisfies (SE1). This proves (iv). Furthermore, (iv) $\Longrightarrow$ (iii) is trivial.

As for (iii) $\Longrightarrow$ (i) assume that (SE1) holds in $\mathcal{K}$. The first task is to show that the composite $X \circ Y$ for $X, Y \in \mathcal{K}$ can be obtained as a conformal composite (supremum) of $X$ with members $Y^{(f)}$ of $\mathcal{K}$, one for each $f \in E \backslash S(X, Y)$. Given such a position $f$, start an 
iteration with $Z^{(\varnothing, f)}:=Y$, and as long as $A \neq S(X, Y)$, apply (SE1) to $X, Z^{(A, f)} \in \mathcal{K}$, which then returns a sign vector

$$
\begin{gathered}
Z^{(A \cup\{e\}, f)} \in \mathcal{W}_{A}\left(X, Z^{(A, f)}\right) \cap \mathcal{K} \subseteq \mathcal{W}_{A}(X, Y) \cap \mathcal{K} \text { with } \\
Z_{e}^{(A \cup\{e\}, f)}=0 \text { and } Z_{f}^{(A \cup\{e\}, f)}=\left(X \circ Z^{(A, f)}\right)_{f}=(X \circ Y)_{f} .
\end{gathered}
$$

In particular, $Z^{(A \cup\{e\}, f)} \in \mathcal{W}_{A \cup\{e\}}(X, Y) \cap \mathcal{K}$. Eventually, the iteration stops with

$$
\begin{aligned}
& Y^{(f)}:=Z^{(S(X, Y), f)} \in \mathcal{W}_{\infty}(X, Y) \cap \mathcal{K} \text { satisfying } \\
& Y_{f}^{(f)}=(X \circ Y)_{f} \text { and }\left(X \circ Y^{(f)}\right)_{e}=X_{e} \text { for all } e \text { separating } X \text { and } Y .
\end{aligned}
$$

Now take the supremum of $X$ and all $Y^{(f)}$ : then

$$
X \circ Y=X \odot \bigodot_{f \in E \backslash S(X, Y)} Y^{(f)}
$$

constitutes the desired representation.

Next consider a composition $X \circ X^{(1)} \circ \ldots \circ X^{(n)}$ of $n+1 \geq 3$ sign vectors from $\mathcal{K}$. By induction on $n$ we may assume that

$$
X^{(1)} \circ \ldots \circ X^{(n)}=Y^{(1)} \odot \ldots \odot Y^{(m)}
$$

where $Y^{(i)} \in \mathcal{K}$ for all $i=1, \ldots, m$. Since any supremum in $\{ \pm 1,0\}^{E}$ needs at most \#E constituents, we may well choose $m=\# E$. Similarly, as the case $n=1$ has been dealt with, each $X \circ Y^{(i)}$ admits a commutative representation

$$
X \circ Y^{(i)}=X \odot Z^{(m(i-1)+1)} \odot Z^{(m(i-1)+2)} \odot \ldots \odot Z^{(m i)}(i=1, \ldots, m) .
$$

We claim that $Z^{(j)}$ and $Z^{(k)}$ commute for all $j, k \in\left\{1, \ldots, m^{2}\right\}$. Indeed,

$$
Z^{(j)} \leq X \circ Y^{(h)} \text { and } Z^{(k)} \leq X \circ Y^{(i)} \text { for some } h, i \in\{1, \ldots, m\} \text {. }
$$

Then

$$
Z^{(j)}, Z^{(k)} \leq\left(X \circ Y^{(h)}\right) \circ Y^{(i)}=\left(X \circ Y^{(i)}\right) \circ Y^{(h)}
$$

because $Y^{(h)}$ and $Y^{(i)}$ commute, whence $Z^{(j)}$ and $Z^{(k)}$ commute as well. Therefore

$$
\begin{aligned}
X \circ X^{(1)} \circ \ldots \circ X^{(n)} & =X \circ Y^{(1)} \circ \ldots \circ Y^{(m)}=\left(X \circ Y^{(1)}\right) \circ \ldots \circ\left(X \circ Y^{(m)}\right) \\
& =X \odot Z^{(1)} \odot \ldots \odot Z^{\left(m^{2}\right)}
\end{aligned}
$$

gives the required representation. We conclude that $(E, \mathcal{L})$ satisfies $(\mathrm{C})$.

To establish (SE1) for $\mathcal{L}$, let $X=X^{(1)} \odot \cdots \odot X^{(n)}$ and $Y=Y^{(1)} \odot \cdots \odot Y^{(m)}$ with $X^{(i)}, Y^{(j)} \in \mathcal{K}$ for all $i, j$. Take $e, f \in E$ such that $e$ separates $X$ and $Y$ and $f$ does not. We may assume that $X_{e}^{(i)}=X_{e}$ for $1 \leq i \leq h, Y_{e}^{(j)}=Y_{e}$ for $1 \leq j \leq k$, and equal to zero otherwise (where $h, k \geq 1$ ). Since $\mathcal{K}$ satisfies (SE1) there exists $Z^{(i, j)} \in \mathcal{W}\left(X^{(i)}, Y^{(j)}\right) \cap \mathcal{K}$ such that $Z_{e}^{(i, j)}=0$ and $Z_{f}^{(i, j)}=\left(X^{(i)} \circ Y^{(j)}\right)_{f}$ for $i \leq h$ and $j \leq k$. Then the composition of all $Z^{(i, 1)}$ for $i \leq h, X^{(i)}$ for $i>h$ and all $Z^{(1, j)}$ for $j \leq k, Y^{(j)}$ for $j>k$ yields the required sign vector $Z \in \mathcal{W}(X, Y)$ with $Z_{e}=0$ and $Z_{f}=(X \circ Y)_{f}$. We conclude that $(E, \mathcal{L})$ is indeed a strong elimination system by Lemma 5 . 
From the preceding proof of (iv) $\Longrightarrow$ (i) we infer that the (supremum-)irreducibles of a strong elimination system $(E, \mathcal{L})$ are a fortiori irreducible with respect to arbitrary composition.

\section{Cocircuits of COMs}

In an OM, a cocircuit is a support-minimal non-zero covector, and the cocircuits form the unique minimal generating system for the entire set of covectors provided that composition over an empty index set is allowed. Thus, in our context the zero vector would have to be added to the generating set, i.e., we would regard it as a cocircuit as well. The cocircuits of COMs that we will consider next should on the one hand generate the entire system and on the other hand their restriction to any maximal face should be the set of cocircuits of the oriented matroid corresponding to that face via Lemma 4 .

For any $\mathcal{K}$ with $\mathcal{J}=\mathcal{J}(\mathcal{L}) \subseteq \mathcal{K} \subseteq \mathcal{L}$ denote by $\operatorname{Min}(\mathcal{K})$ the set of all minimal sign vectors of $\mathcal{K}$. Clearly, $\operatorname{Min}(\odot \mathcal{K})=\operatorname{Min}(\mathcal{K})=\operatorname{Min}(\mathcal{J})$. We say that $Y$ covers $X$ in $\mathcal{L}=\odot \mathcal{J}$ (in symbols: $X \prec Y$ ) if $X<Y$ holds and there is no sign vector $Z \in \mathcal{L}$ with $X<Z<Y$. The following set $\mathcal{C}$ is intermediate between $\mathcal{J}$ and $\mathcal{L}$ :

$$
\mathcal{C}=\mathcal{C}(\mathcal{L}):=\mathcal{J}(\mathcal{L}) \cup\{X \in \mathcal{L}: W \prec X \text { for some } W \in \operatorname{Min}(\mathcal{L})\} .
$$

Since $\operatorname{Min}(\mathcal{L})=\operatorname{Min}(\mathcal{J})$ and every cover $X \notin \mathcal{J}$ of some $W \in \operatorname{Min}(\mathcal{J})$ is above some other $V \in \operatorname{Min}(\mathcal{J})$, we obtain:

$$
\mathcal{C}=\mathcal{C}(\mathcal{J}):=\mathcal{J} \cup\{W \odot V: V, W \in \operatorname{Min}(\mathcal{J}) \text { and } W \prec W \odot V\} .
$$

We will make use of the following variant of face symmetry restricted to comparable covectors:

$(\mathbf{F S} \leq) \quad X \circ-Y \in \mathcal{L}$ for all $X \leq Y$ in $\mathcal{L}$.

Note that $(\mathrm{FS})$ and $(\mathrm{FS} \leq)$ are equivalent in any system $\mathcal{L}$ satisfying $(\mathrm{C})$, as one can let $X \circ Y$ substitute $Y$ in (FS). We can further weaken face symmetry by restricting it to particular covering pairs $X \prec Y$ :

$\left(\mathbf{F S}^{\prec}\right) W \circ-Y \in \mathcal{L}$ for all $W \in \operatorname{Min}(\mathcal{L})$ and $Y \in \mathcal{L}$ with $W \prec Y$ in $\mathcal{L}$, or equivalently, $W \circ-Y \in \mathcal{C}$ for all $W \in \operatorname{Min}(\mathcal{C})$ and $Y \in \mathcal{C}$ with $W \prec Y$.

Indeed, since sign reversal constitutes an order automorphism of $\{ \pm 1,0\}^{E}$, we readily infer that in $\left(\mathrm{FS}^{\prec}\right) W \circ-Y$ covers $W$, for if there was $X \in \mathcal{L}$ with $W \prec X<W \circ-Y$, then $W<W \circ-X<W \circ-(W \circ-Y)=W \circ Y=Y$, a contradiction.

To show that $\left(\mathrm{FS}^{\prec}\right)$ implies $(\mathrm{FS} \leq)$ takes a little argument, as we will see next.

Proposition 5. Let $(E, \mathcal{J})$ be a system of sign vectors. Then $(E, \odot \mathcal{J})$ is a COM such that $\mathcal{J}$ is its set of irreducibles if and only if $\mathcal{C}=\mathcal{C}(\mathcal{J})$ satisfies $\left(\mathrm{FS}^{\prec}\right)$ and $\mathcal{J}$ satisfies $(\mathrm{SE} 1)$ and (IRR) if $X=\bigodot_{i=1}^{n} X_{i}$ for $X, X_{1}, \ldots, X_{n} \in \mathcal{J}(n \geq 2)$, then $X=X_{i}$ for some $1 \leq i \leq n$.

Proof. First, assume that $(E, \mathcal{L}=\bigodot \mathcal{J})$ is a COM with $\mathcal{J}=\mathcal{J}(\mathcal{L})$. From Theorem 1 we know that $\mathcal{J}$ satisfies (SE1), while (IRR) just expresses irreducibility. Since $\mathcal{L}$ is the set of 
covectors of a COM, from the discussion preceding the theorem it follows that $\mathcal{L}$ satisfies $\left(\mathrm{FS}^{\prec}\right)$. Consequently, $\mathcal{C}=\mathcal{C}(\mathcal{J})$ satisfies $\left(\mathrm{FS}^{\prec}\right)$.

Conversely, in the light of Theorem 1 , it remains to prove that $\left(\mathrm{FS}^{\prec}\right)$ for $\mathcal{C}$ implies $(\mathrm{FS} \leq)$ for $(E, \mathcal{L})$. Note that for $W<X<Y$ in $\mathcal{L}$ we have $X \circ-Y=X \circ W \circ-Y$, whence for $W<Y \in \mathcal{L}$ we only need to show $W \circ-Y \in \mathcal{L}$ when $W$ is a minimal sign vector of $\mathcal{L}$ (and thus belonging to $\mathcal{J} \subseteq \mathcal{C}$ ). Now suppose that $W \circ-Y \notin \mathcal{L}$ for some covector $Y$ such that $\# Y^{0}$ is as large as possible. Thus as $Y \notin \mathcal{C}$ there exists $X \in \mathcal{L}$ with $W \prec X<Y$. By (FS $\left.{ }^{\prec}\right)$, $W \circ-X \in \mathcal{L}$ holds. Pick any element $e \in S(W \circ-X \circ Y, Y)=W^{0} \cap \underline{X}$ and choose some $Z \in \mathcal{L}$ with $Z_{e}=0$ and $Z_{f}=(W \circ-X \circ Y)_{f}$ for all $f \in E \backslash S(W \circ-X \circ Y, Y)$ by virtue of (SE). In particular, $Y=X \circ Z$. Then necessarily $W<Z$ and $Y^{0} \cup\{e\} \subseteq Z^{0}$, so that $W \circ-Z \in \mathcal{L}$ by the maximality hypothesis. Therefore with Theorem 1 we get

$$
W \circ-Y=W \circ-(X \circ Z)=(W \circ-X) \circ(W \circ-Z) \in \mathcal{L},
$$

which is a contradiction. This establishes $(\mathrm{FS} \leq)$ for $\mathcal{L}$ and thus completes the proof of Proposition 5 .

Proposition 5 yields the following alternative axiomatization of COMs in terms of covectors, that is of independent interest:

Corollary 1. A system $(E, \mathcal{L})$ of sign vectors is a COM if and only if $(E, \mathcal{L})$ satisfies $(\mathrm{CC})$, $(\mathrm{SE} 1)$ and $\left(\mathrm{FS}^{\prec}\right)$.

Let us now advance towards the axiomatization of COMs in terms of cocircuits. Given a $\operatorname{COM}(E, \mathcal{L})$, we call the minimal sign vectors of $\mathcal{L}$ the improper cocircuits of $(E, \mathcal{L})$. A proper cocircuit is any sign vector $Y \in \mathcal{L}$ which covers some improper cocircuit $X$. Cocircuit then refers to either kind, improper or proper. Hence, $\mathcal{C}(\mathcal{L})$ is the set of all cocircuits of $\mathcal{L}$. Note that in oriented matroids the zero vector is the only improper cocircuit and the usual OM cocircuits are the proper cocircuits in our terminology. In lopsided systems $(E, \mathcal{L})$, the improper cocircuits are the barycenters of maximal hypercubes [4]. In a COM improper circuits are irreducible, but not all proper circuits need to be irreducible. Here is the main result of this section.

Theorem 2. Let $(E, \mathcal{C})$ be a system of sign vectors and let $\mathcal{L}:=\bigodot \mathcal{C}$. Then $(E, \mathcal{L})$ is a $C O M$ such that $\mathcal{C}$ is its set of cocircuits if and only if $\mathcal{C}$ satisfies $(\mathrm{SE} 1),\left(\mathrm{FS}^{\prec}\right)$, and

$(\mathbf{C O C}) \mathcal{C}=\operatorname{Min}(\mathcal{C}) \cup\{Y \in \bigodot \mathcal{C}: W \prec Y$ for some $W \in \operatorname{Min}(\mathcal{C})\}$.

Proof. Let $(E, \mathcal{L})$ be a COM and $\mathcal{C}$ be its set of cocircuits. By Proposition $5, \mathcal{C}$ satisfies $\left(\mathrm{FS}^{\prec}\right)$. From the proof of Theorem 1, part (ii) $\Rightarrow$ (iv), we know that a sign vector $Z$ demanded in (SE1) could always be chosen from the irreducibles, which are particular cocircuits. Therefore $\mathcal{C}=\mathcal{C}(L)$ satisfies (SE1). Finally, (COC) just expresses that $\mathcal{C}$ exactly comprises the cocircuits of the set $\mathcal{L}$ it generates.

Conversely, $\mathcal{L}$ satisfies $(\mathrm{CC})$ by definition. Since $\mathcal{J}(\mathcal{L}) \subseteq \mathcal{C}$ and $\mathcal{C}$ satisfies (SE1), applying Theorem 1 we conclude that $\mathcal{J}$ satisfies (SE1). Consequently, as $\mathcal{C}$ satisfies $\left(\mathrm{FS}^{\prec}\right.$ ) and $\mathcal{J}$ satisfies $(\mathrm{SE} 1), \mathcal{L}$ is a $\mathrm{COM}$ by virtue of Proposition 5. 
To give a simple class of planar examples, consider the hexagonal grid, realized as the 1-skeleton of the regular tiling of the plane with (unit) hexagons. A benzenoid graph is the 2-connected graph formed by the vertices and edges from hexagons lying entirely within the region bounded by some cycle in the hexagonal grid; the given cycle thus constitutes the boundary of the resulting benzenoid graph [25. A cut segment is any minimal (closed) segment of a line perpendicular to some edge and passing through its midpoint such that the removal of all edges cut by the segment results in exactly two connected components, one signed + and the other - . The ground set $E$ comprises all these cut segments. The set $\mathcal{L}$ then consists of all sign vectors corresponding to the vertices and the barycenters (midpoints) of edges and 6-cycles (hexagons) of this benzenoid graph. For verifying that $(E, \mathcal{L})$ actually constitutes a COM, it is instructive to apply Proposition 5: the set $\mathcal{J}$ of irreducible members of $\mathcal{L}$ encompasses the barycenter vectors of the boundary edges and of all hexagons of the benzenoid. The barycenter vectors of two hexagons/edges/vertices are sign consistent exactly when they are incident. Therefore $\mathcal{J}$ generates all covectors of $\mathcal{L}$ via $(\mathrm{CC})$. Condition $\left(\mathrm{FS}^{\prec}\right)$ is realized through inversion of an edge at the center of a hexagon it is incident with. Condition (SE1) is easily checked by considering two cases each (depending on whether $Z$ is eventually obtained as a barycenter vector of a hexagon or of an edge) for pairs $X, Y$ of hexagon/edge barycenters.

\section{Hyperplanes, CARRIERs, AND halfspaCeS}

For a system $(E, \mathcal{L})$ of sign vectors, a hyperplane of $\mathcal{L}$ is the set

$$
\mathcal{L}_{e}^{0}:=\left\{X \in \mathcal{L}: X_{e}=0\right\} \text { for some } e \in E .
$$

The carrier $N\left(\mathcal{L}_{e}^{0}\right)$ of the hyperplane $\mathcal{L}_{e}^{0}$ is the union of all faces $F\left(X^{\prime}\right)$ of $\mathcal{L}$ with $X^{\prime} \in \mathcal{L}_{e}^{0}$, that is,

$$
N\left(\mathcal{L}_{e}^{0}\right):=\left\{X \in \mathcal{L}: W \leq X \text { for some } W \in \mathcal{L}_{e}^{0}\right\} .
$$

The positive and negative ("open") halfspaces supported by the hyperplane $\mathcal{L}_{e}^{0}$ are

$$
\begin{aligned}
& \mathcal{L}_{e}^{+}:=\left\{X \in \mathcal{L}: X_{e}=+1\right\}, \\
& \mathcal{L}_{e}^{-}:=\left\{X \in \mathcal{L}: X_{e}=-1\right\} .
\end{aligned}
$$

The carrier $N\left(\mathcal{L}_{e}^{0}\right)$ minus $\mathcal{L}_{e}^{0}$ splits into its positive and negative parts:

$$
\begin{aligned}
& N^{+}\left(\mathcal{L}_{e}^{0}\right):=\mathcal{L}_{e}^{+} \cap N\left(\mathcal{L}_{e}^{0}\right), \\
& N^{-}\left(\mathcal{L}_{e}^{0}\right):=\mathcal{L}_{e}^{-} \cap N\left(\mathcal{L}_{e}^{0}\right) .
\end{aligned}
$$

The closure of the disjoint halfspaces $\mathcal{L}_{e}^{+}$and $\mathcal{L}_{e}^{-}$just adds the corresponding carrier:

$$
\begin{aligned}
& \overline{\mathcal{L}_{e}^{+}}:=\mathcal{L}_{e}^{+} \cup N\left(\mathcal{L}_{e}^{0}\right)=\mathcal{L}_{e}^{+} \cup \mathcal{L}_{e}^{0} \cup N^{-}\left(\mathcal{L}_{e}^{0}\right), \\
& \overline{\mathcal{L}_{e}^{-}}:=\mathcal{L}_{e}^{-} \cup N\left(\mathcal{L}_{e}^{0}\right)=\mathcal{L}_{e}^{-} \cup \mathcal{L}_{e}^{0} \cup N^{+}\left(\mathcal{L}_{e}^{0}\right) .
\end{aligned}
$$

The former is called the closed positive halfspace supported by $\mathcal{L}_{e}^{0}$, and the latter is the corresponding closed negative halfspace. Both overlap exactly in the carrier. 


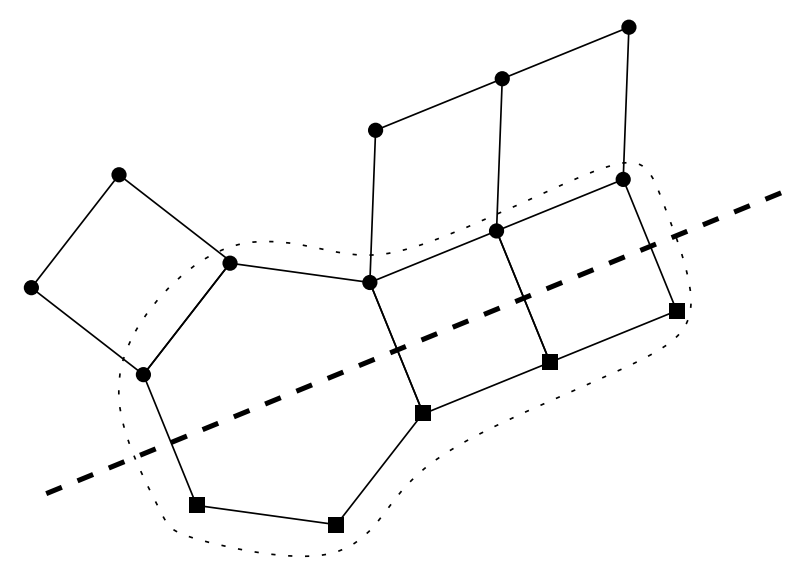

FiguRE 3. A hyperplane (dashed), its associated open halfspaces (square and round vertices, respectively) and the associated carrier (dotted) in a COM.

Proposition 6. Let $(E, \mathcal{L})$ be a system of sign vectors. Then all its hyperplanes, its carriers and their positive and negative parts, its halfspaces and their closures are strong elimination systems or $C O M s$, respectively, whenever $(E, \mathcal{L})$ is such. If $(E, \mathcal{L})$ is an $O M$, then so are all its hyperplanes and carriers.

Proof. We already know that fibers preserve (C),(FS), and (SE). Moreover, intersections preserve the two properties (C) and (FS). Since $X^{\prime} \leq X$ and $Y^{\prime} \leq Y$ imply both $X^{\prime} \leq$ $X \circ Y, X^{\prime} \leq X \circ(-Y)$ and $Y^{\prime} \leq Y \circ X, Y^{\prime} \leq Y \circ(-X)$, we infer that (C) and (FS) carry over from $\mathcal{L}$ to $N\left(\mathcal{L}_{e}^{0}\right)$

In what follows let $(E, \mathcal{L})$ be a strong elimination system. We need to show that $N\left(\mathcal{L}_{e}^{0}\right)$ satisfies (SE). Let $X^{\prime}, Y^{\prime} \in \mathcal{L}_{e}^{0}$ and $X, Y \in \mathcal{L}$ such that $X^{\prime} \leq X$ and $Y^{\prime} \leq Y$. Then $S\left(X^{\prime}, Y^{\prime}\right) \subseteq S(X, Y)$. Apply (SE) to the pair $X, Y$ in $\mathcal{L}$ relative to some element $e^{\prime}$ separating $X$ and $Y$. This yields some $Z \in \mathcal{W}(X, Y)$ with $Z_{e^{\prime}}=0$ and $Z_{f}=(X \circ Y)_{f}$ for all $f \in E \backslash$ $S(X, Y)$. If $e^{\prime} \in S\left(X^{\prime}, Y^{\prime}\right)$ as well, then apply (SE) to $X^{\prime}, Y^{\prime}$ in $\mathcal{L}_{e}^{0}$ giving $Z^{\prime} \in \mathcal{W}\left(X^{\prime}, Y^{\prime}\right) \cap \mathcal{L}_{e}^{0}$ with $Z_{e^{\prime}}^{\prime}=0$ and $Z_{f}^{\prime}=\left(X^{\prime} \circ Y^{\prime}\right)_{f}$ for all $f \in E \backslash S(X, Y) \subseteq E \backslash S\left(X^{\prime}, Y^{\prime}\right)$. If $e^{\prime} \in \underline{X}^{\prime} \backslash \underline{Y}^{\prime}$, then put $Z^{\prime}:=Y^{\prime}$. Else, if $e^{\prime} \in E \backslash \underline{X}^{\prime}$, put $Z^{\prime}:=X^{\prime}$. Observe that all cases are covered as $S\left(X^{\prime}, Y^{\prime}\right)=S(X, Y) \cap \underline{X}^{\prime} \cap \underline{Y}^{\prime}$. We claim that in any case $Z^{\prime} \circ Z$ is the required sign vector fulfilling (SE) for $X, Y$ relative to $e^{\prime}$. Indeed, $Z^{\prime} \circ Z$ belongs to $N\left(\mathcal{L}_{e}^{0}\right)$ since $Z^{\prime} \in \mathcal{L}_{e}^{0}$ and $Z \in \mathcal{L}$. Then $Z^{\prime} \circ Z \in \mathcal{W}(X, Y)$ because $\mathcal{W}\left(X^{\prime}, Y^{\prime}\right) \subseteq \mathcal{W}(X, Y)$ and $\mathcal{W}(X, Y)$ is closed under composition. Let $f \in E \backslash S(X, Y)$. Then $X_{f}^{\prime}, X_{f}, Y_{f}^{\prime}, Y_{f}$ all commute.

In particular,

$$
\left(Z^{\prime} \circ Z\right)_{f}=Z_{f}^{\prime} \circ Z_{f}=X_{f}^{\prime} \circ Y_{f}^{\prime} \circ X_{f} \circ Y_{f}=X_{f}^{\prime} \circ X_{f} \circ Y_{f}^{\prime} \circ Y_{f}=(X \circ Y)_{f}
$$

whenever both $Y_{e^{\prime}}^{\prime}=Y_{e^{\prime}}$ and $X_{e^{\prime}}^{\prime}=X_{e^{\prime}}$ hold. If however $Y_{e}^{\prime}=0$, then $\left(Y^{\prime} \circ Z\right)_{f}=Y_{f}^{\prime} \circ X_{f} \circ Y_{f}=$ $X_{f} \circ Y_{f}^{\prime} \circ Y_{f}=(X \circ Y)_{f}$. Else, if $X_{e}^{\prime}=0$, then $\left(X^{\prime} \circ Z\right)_{f}=X_{f}^{\prime} \circ X_{f} \circ Y_{f}=(X \circ Y)_{f}$. This finally shows that the carrier of $\mathcal{L}_{e}^{0}$ satisfies $(\mathrm{SE})$. 
To prove that $\overline{\mathcal{L}_{e}^{+}}$satisfies (SE) for a pair $X, Y \in \overline{\mathcal{L}_{e}^{+}}$relative to some $e^{\prime} \in S(X, Y)$, assume that $X \in \mathcal{L}_{e}^{+}$and $Y \in N\left(\mathcal{L}_{e}^{0}\right) \backslash \mathcal{L}_{e}^{+}$since (SE) has already been established for both $\mathcal{L}_{e}^{+}$and $N\left(\mathcal{L}_{e}^{0}\right)$ and the required sign vector would equally serve the pair $Y, X$. Now pick any $Y^{\prime} \in \mathcal{L}_{e}^{0}$ with $Y^{\prime} \leq Y$. Then two cases can occur for $e^{\prime} \in S(X, Y)$.

Case 1. $Y_{e^{\prime}}^{\prime}=0$.

Then $\left(Y^{\prime} \circ X\right)_{e^{\prime}}=X_{e^{\prime}}, S\left(Y^{\prime} \circ X, Y\right) \subseteq S(X, Y)$, and $Y^{\prime} \leq Y^{\prime} \circ X$, whence $Y^{\prime} \circ X \in N\left(\mathcal{L}_{e}^{0}\right)$. Applying (SE) to $Y^{\prime} \circ X, Y$ in $N\left(\mathcal{L}_{e}^{0}\right)$ relative to $e^{\prime}$ yields $Z^{\prime} \in \mathcal{W}\left(Y^{\prime} \circ X, Y\right) \subseteq \mathcal{W}(X, Y)$ with $Z_{e^{\prime}}^{\prime}=0$ and

$$
Z_{f}^{\prime}=Y_{f}^{\prime} \circ X_{f} \circ Y_{f}=X_{f} \circ Y_{f}^{\prime} \circ Y_{f}=(X \circ Y)_{f} \text { for all } f \in E \backslash S(X, Y) .
$$

Case 2. $Y_{e^{\prime}}^{\prime}=Y_{e^{\prime}}$.

As above we can select $Z \in \mathcal{W}(X, Y)$ with $Z_{e^{\prime}}=0$ and $Z_{f}=(X \circ Y)_{f}$ for all $f \in$ $E \backslash S(X, Y)$. Analogously choose $Z^{\prime} \in \mathcal{W}\left(X, Y^{\prime}\right)$ with $Z_{e^{\prime}}^{\prime}=0$ and $Z_{f}^{\prime}=\left(X \circ Y^{\prime}\right)_{f}$ for all $f \in E \backslash S\left(X, Y^{\prime}\right)$. We claim that in this case $Z^{\prime} \circ Z$ is a sign vector from $\mathcal{L}_{e}^{+}$as required for $X, Y$ relative to $e^{\prime}$. Indeed, $Z_{e}^{\prime}=\left(X \circ Y^{\prime}\right)_{e}=+1=(X \circ Y)_{e}$ because $X_{e}=+1, Y_{e}^{\prime}=0$ and consequently $e \notin S\left(X, Y^{\prime}\right)$. For $f \in E \backslash S(X, Y)$ we have

$$
\left(Z^{\prime} \circ Z\right)_{f}=X_{f} \circ Y_{f}^{\prime} \circ X_{f} \circ Y_{f}=X_{f} \circ X_{f} \circ Y_{f}^{\prime} \circ Y_{f}=(X \circ Y)_{f}
$$

by commutativity, similarly as above. This proves that $\overline{\mathcal{L}_{e}^{+}}$satisfies $(\mathrm{SE})$.

To show that $N^{+}\left(\mathcal{L}_{e}^{0}\right)=\mathcal{L}_{e}^{+} \cap N\left(\mathcal{L}_{e}^{0}\right)$ satisfies (SE), we can apply (SE) to some pair $X, Y \in N^{+}\left(\mathcal{L}_{e}^{0}\right)$ relative to some $e^{\prime} \in S(X, Y)$ first within $N\left(\mathcal{L}_{e}^{0}\right)$ and then within $\mathcal{L}_{e}^{+}$to obtain two sign vectors $Z^{\prime} \in N\left(\mathcal{L}_{e}^{0}\right) \cap \mathcal{W}(X, Y)$ and $Z \in \mathcal{L}_{e}^{+} \cap \mathcal{W}(X, Y)$ such that $Z_{e^{\prime}}^{\prime}=0=Z_{e^{\prime}}$ and $Z_{f}^{\prime}=(X \circ Y)_{f}=Z_{f}$ for all $f \in E \backslash S(X, Y)$. Then $Z^{\prime} \leq Z^{\prime} \circ Z \in N\left(\mathcal{L}_{e}^{0}\right)$ and $\left(Z^{\prime} \circ Z\right)_{e}=$ $(X \circ Y)_{e}=+1$ as $e \notin S(X, Y)$. Moreover, $\left(Z^{\prime} \circ Z\right)_{f}=(X \circ Y)_{f}$ for all $f \in E \backslash S(X, Y)$. This establishes (SE) for $N^{+}\left(\mathcal{L}_{e}^{0}\right)$. The proofs for $\overline{\mathcal{L}_{e}^{-}}$and $N^{-}\left(\mathcal{L}_{e}^{0}\right)$ are completely analogous. The last statement of the proposition is then trivially true because the zero vector, once present in $\mathcal{L}$, is also contained in all hyperplanes (and hence the carriers).

A particular class of COMs obtained by the above proposition are halfspaces of OMs. These are usually called affine oriented matroids, see [27] and [10, p. 154]. Karlander [27] has shown how an OM can be reconstructed from any of its halfspaces. The proof of his intriguing axiomatization of affine oriented matroids, however, has a gap, which has been filled only recently [5]. Only few results exist about the complex given by an affine oriented matroid $[18,19$.

We continue with the following recursive characterization of COMs:

Theorem 3. Let $(E, \mathcal{L})$ be a semisimple system of sign vectors. Then $(E, \mathcal{L})$ is a strong elimination system if and only if the following four requirements are met:

$(1)$ the composition rule $(C)$ holds in $(E, \mathcal{L})$,

(2) all hyperplanes of $(E, \mathcal{L})$ are strong elimination systems,

(3) the tope graph of $(E, \mathcal{L})$ is a partial cube, 
(4) for each pair $X, Y$ of adjacent topes (i.e., with $\# S(X, Y)=1$ ) the barycenter of the corresponding edge, i.e. the sign vector $\frac{1}{2}(X+Y)$, belongs to $\mathcal{L}$.

Moreover, $(E, \mathcal{L})$ is a COM if and only if it satisfies (1),(3),(4), and

$\left(2^{\prime}\right)$ all hyperplanes of $(E, \mathcal{L})$ are $C O M s$,

In particular, $(E, \mathcal{L})$ is an $O M$ if and only if it satisfies (1),(4), and

$\left(2^{\prime \prime}\right)$ all hyperplanes of $(E, \mathcal{L})$ are $O M s$,

$\left(3^{\prime}\right)$ the tope set of $(E, \mathcal{L})$ is a simple acycloid, see [26], i.e., induces a partial cube and satisfies (Sym).

Proof. The "if" directions of all three assertions directly follow from Propositions 6 and 2 Conversely, using (1) and Lemma 5 , we only need to verify $\left(\mathrm{SE}^{=}\right)$to prove the first assertion.

To establish $\left(\mathrm{SE}^{=}\right)$, let $X$ and $Y$ be any different sign vectors from $\mathcal{L}$. Assume that $\underline{X}=\underline{Y}$ and $e \in S(X, Y)$. If the supports are not all of $E$, then we can apply ( $\left.\mathrm{SE}^{=}\right)$to the hyperplane associated with a zero coordinate of $X$ and $Y$ according to condition (2) and obtain a sign vector $Z$ as required. Otherwise, both $X$ and $Y$ are topes. Then a shortest path joining $X$ and $Y$ in the tope graph is indexed by the elements of $S(X, Y)$ and thus includes an edge associated with $e$. Then the corresponding barycenter map $Z$ (that belongs to $\mathcal{L}$ by condition $(4))$ of this edge does the job. Thus $(E, \mathcal{L})$ is a semisimple strong elimination system.

In order to complete the proof of the second assertion it remains to establish (FS $\leq$ ). So let $X$ and $Y$ be any different sign vectors from $\mathcal{L}$ with $X \circ Y=Y$. In particular, $X$ is not a tope and $Y$ belongs to the face $F(X)$. If the support $\underline{Y}$ does not equal $E$, then again we find a common zero coordinate of $X$ and $Y$, so that we can apply $\left(\mathrm{FS}^{\leq} \leq\right)$in the corresponding hyperplane to yield the sign vector opposite to $Y$ relative to $X$. So we may assume that $Y$ is a tope. Since $(E, \mathcal{L})$ is a semisimple strong elimination system, from Proposition 2 we infer that the tope graph of $F(X)$ is a partial cube containing at least two topes. Thus there exists a tope $U \in F(X)$ adjacent to $Y$ in the tope graph, say $S(U, Y)=\{e\}$. Let $W$ be the barycenter map of this edge. Applying $(\mathrm{FS} \leq)$ for the pair $X, W$ in the hyperplane $\mathcal{L}_{e}^{0}$ relative to $e$ we obtain $X \circ(-W) \in \mathcal{L}_{e}^{0}$. By (1) we have $X \circ(-W) \circ U \in \mathcal{L}$. Since $X \circ(-W) \circ U=X \circ(-Y)$ this concludes the proof.

As for the third assertion, note that symmetric COMs are OMs and symmetry for nontopes is implied by symmetry for hyperplanes.

\section{Decomposition And amalgamation}

Proposition 6 provides the necessary ingredients for a decomposition of a COM, which is not an $\mathrm{OM}$, into smaller $\mathrm{COM}$ constituents. Assume that $(E, \mathcal{L})$ is a semisimple $\mathrm{COM}$ that is not an OM. Put $\mathcal{L}^{\prime}:=\mathcal{L}_{e}^{-}$and $\mathcal{L}^{\prime \prime}:=\overline{\mathcal{L}_{e}^{+}}$. Then $\mathcal{L}=\mathcal{L}^{\prime} \cup \mathcal{L}^{\prime \prime}$ and $\mathcal{L}^{\prime} \cap \mathcal{L}^{\prime \prime}=N^{-}\left(\mathcal{L}_{e}^{0}\right)$. Since $X$ determines a maximal face not included in $\mathcal{L}_{e}^{0}$, we infer that $\mathcal{L}^{\prime} \backslash \mathcal{L}^{\prime \prime} \neq \varnothing$ and trivially $\mathcal{L}^{\prime \prime} \backslash \mathcal{L}^{\prime} \neq \varnothing$. By Proposition 6, all three systems $\left(E, \mathcal{L}^{\prime}\right),\left(E, \mathcal{L}^{\prime \prime}\right)$, and $\left(E, \mathcal{L}^{\prime} \cap \mathcal{L}^{\prime \prime}\right)$ are COMs, which are easily seen to be semisimple. 
Moreover, $\mathcal{L}^{\prime} \circ \mathcal{L}^{\prime \prime} \subseteq \mathcal{L}^{\prime}$ holds trivially. If $W \in \mathcal{L}_{e}^{0}$ and $X \in \mathcal{L}_{e}^{-}$, then $W \circ X \in F(W) \subseteq$ $N\left(\mathcal{L}_{e}^{0}\right)$, whence $\mathcal{L}^{\prime \prime} \circ \mathcal{L}^{\prime} \subseteq \mathcal{L}^{\prime \prime}$. This motivates the following amalgamation process which in a way reverses this decomposition procedure.

We say that a system $(E, \mathcal{L})$ of sign vectors is a $C O M$ amalgam of two semisimple COMs $\left(E, \mathcal{L}^{\prime}\right)$ and $\left(E, \mathcal{L}^{\prime \prime}\right)$ if the following conditions are satisfied:

(1) $\mathcal{L}=\mathcal{L}^{\prime} \cup \mathcal{L}^{\prime \prime}$ with $\mathcal{L}^{\prime} \backslash \mathcal{L}^{\prime \prime}, \mathcal{L}^{\prime \prime} \backslash \mathcal{L}^{\prime}, \mathcal{L}^{\prime} \cap \mathcal{L}^{\prime \prime} \neq \varnothing$

(2) $\left(E, \mathcal{L}^{\prime} \cap \mathcal{L}^{\prime \prime}\right)$ is a semisimple $\mathrm{COM}$;

(3) $\mathcal{L}^{\prime} \circ \mathcal{L}^{\prime \prime} \subseteq \mathcal{L}^{\prime}$ and $\mathcal{L}^{\prime \prime} \circ \mathcal{L}^{\prime} \subseteq \mathcal{L}^{\prime \prime}$

(4) for $X \in \mathcal{L}^{\prime} \backslash \mathcal{L}^{\prime \prime}$ and $Y \in \mathcal{L}^{\prime \prime} \backslash \mathcal{L}^{\prime}$ with $X^{0}=Y^{0}$ there exists a shortest path in the graphical hypercube on $\{ \pm 1\}^{E \backslash X^{0}}$ for which all its vertices and barycenters of its edges belong to $\mathcal{L} \backslash X^{0}$.

Proposition 7. The COM amalgam of semisimple COMs $\left(E, \mathcal{L}^{\prime}\right)$ and $\left(E, \mathcal{L}^{\prime \prime}\right)$ constitutes a semisimple $C O M(E, \mathcal{L})$ for which every maximal face is a maximal face of at least one of the two constituents.

Proof. $\mathcal{L}=\mathcal{L}^{\prime} \cup \mathcal{L}^{\prime \prime}$ satisfies $(\mathrm{C})$ because $\mathcal{L}^{\prime}$ and $\mathcal{L}^{\prime \prime}$ do and for $X \in \mathcal{L}^{\prime}$ and $Y \in \mathcal{L}^{\prime \prime}$ one obtains $X \circ Y \in \mathcal{L}^{\prime} \subseteq \mathcal{L}$ and $Y \circ X \in \mathcal{L}^{\prime \prime} \subseteq \mathcal{L}$ by (3). Then $\mathcal{L}$ also satisfies (FS ${ }^{\leq}$) since for $X \leq Y=X \circ Y$ in $\mathcal{L}$ the only nontrivial case is that $X \in \mathcal{L}^{\prime}$ and $Y \in \mathcal{L}^{\prime \prime}$, say. Then $Y=X \circ Y \in \mathcal{L}^{\prime}$ by $(3)$, whence $X \circ-Y \in \mathcal{L}^{\prime} \subseteq \mathcal{L}$.

Every minimal sign vector $X \in \mathcal{L}$, say $X \in \mathcal{L}^{\prime}$, yields the face $F(X)=\{X \circ Y: Y \in \mathcal{L}\} \subseteq$ $\mathcal{L}^{\prime} \circ \mathcal{L} \subseteq \mathcal{L}^{\prime}$. It is evident that $(E, \mathcal{L})$ is semisimple.

By Lemma 5, it remains to show ( $\left.\mathrm{SE}^{=}\right)$for two sign vectors $X$ and $Y$ of $\mathcal{L}$ with $X^{0}=Y^{0}$, where $X \in \mathcal{L}^{\prime} \backslash \mathcal{L}^{\prime \prime}$ and $Y \in \mathcal{L}^{\prime \prime} \backslash \mathcal{L}^{\prime}$. Then let $e \in S(X, Y)$ and $f \in E \backslash S(X, Y)$. Then the barycenter of an e-edge on a shortest path $P$ from $X \backslash X^{0}$ to $Y \backslash X^{0}$ between $\mathcal{L}^{\prime} \backslash X^{0}$ and $\mathcal{L}^{\prime \prime} \backslash X^{0}$ (guaranteed by condition (4)) yields the desired sign vector $Z \in \mathcal{L}$ with $Z_{e}=0$, $X^{0} \subseteq Z^{0}$, and $Z \in \mathcal{W}(X, Y)$. Since $X^{0}=Y^{0}$, we have $X_{f}=Y_{f}$ by the choice of $f$. Since $P$ is shortest, we get $Z_{f}=(X \circ Y)_{f}$.

Summarizing the previous discussion and results, we obtain

Corollary 2. Semisimple COMs are obtained via successive COM amalgamations from their maximal faces (that can be contracted to OMs).

\section{Euler-Poincaré formulae}

In this section, we generalize the Euler-Poincaré formula known for OMs to COMs, which involves the rank function. This is an easy consequence of decomposition and amalgamation. In the case of lopsided systems and their hypercube cells the rank of a cell is simply expressed as the cardinality of the zero set of its associated covector.

Given an $\mathrm{OM}$ of rank $r$, for $0 \leq i \leq r-1$ one defines $f_{i}$ as the number of cells of dimension $f_{i}$ of the corresponding decomposition of the $(r-1)$-sphere, see Section 11 for more about this representation. It is well-known (cf. 10 , Corollary 4.6.11]) that $\sum_{i=0}^{r-1}(-1)^{i} f_{i}=1+(-1)^{r-1}$. 
Adding the summand $(-1)^{-1} f_{-1}=-1$ here artificially yields $\sum_{i=-1}^{r-1}(-1)^{i} f_{i}=(-1)^{r-1}$. Multiplying this equation by $(-1)^{r-1}$ and substituting $i$ by $r-1-j$ yields

$$
\sum_{j=0}^{r}(-1)^{j} f_{r-1-j}=\sum_{i=1}^{r-1}(-1)^{r-1-i} f_{i}=1 .
$$

As $f_{r-1-j}$ gives the number of $\mathrm{OM}$ faces of rank $j$ we can restate this formula in covector notation as $\sum_{X \in \mathcal{L}}(-1)^{r(X)}=1$, where $r(X)$ is the rank of the OM $F(X) \backslash \underline{X}$. We define the rank of the covector of a $\mathrm{COM}$ in the same way.

Since COMs arise from OMs by successive COM amalgamations, which do not create new faces, and at a step from $\mathcal{L}^{\prime}$ and $\mathcal{L}^{\prime \prime}$ to the amalgamated $\mathcal{L}$ each face in the intersection is counted exactly twice, we obtain

$$
\sum_{X \in \mathcal{L}}(-1)^{r(X)}=\sum_{X \in \mathcal{L}^{\prime}}(-1)^{r(X)}+\sum_{X \in \mathcal{L}^{\prime \prime}}(-1)^{r(X)}-\sum_{X \in \mathcal{L}^{\prime} \cap \mathcal{L}^{\prime \prime}}(-1)^{r(X)}=1 .
$$

Proposition 8. Every $\operatorname{COM}(E, \mathcal{L})$ satisfies the Euler-Poincaré formula $\sum_{X \in \mathcal{L}}(-1)^{r(X)}=1$.

We now characterize lopsided systems in terms of an Euler-Poincaré formula. A system $(E, \mathcal{L})$ is said to satisfy the Euler-Poincaré formula for zero sets if

$$
\sum_{X \in \mathcal{L}}(-1)^{\# X^{0}}=1
$$

Proposition 9. The following assertions are equivalent for a system $(E, \mathcal{L})$ :

(i) $(E, \mathcal{L})$ is lopsided, that is, $(E, \mathcal{L})$ is a COM satisfying $(I C)$;

(ii) 38 every topal fiber of $(E, \mathcal{L})$ satisfies the Euler formula for zero sets, and $\mathcal{L}$ is determined by the topes in the following way: for each sign vector $X \in\{ \pm 1,0\}^{E}$, $X \in \mathcal{L} \Rightarrow X \circ Y \in \mathcal{L}$ for all $Y \in\{ \pm 1\}^{E}$

(iii) every contraction of a topal fiber of $(E, \mathcal{L})$ satisfies the Euler formula for zero sets in its own right.

Proof. Deletions, contractions, and fibers of lopsided sets are COMs satisfying (IC) as well, that is, are again lopsided. In case of a lopsided system $(E, \mathcal{L})$ for every $X \in \mathcal{L}$ we have $r(X)=\# X^{0}$. Therefore by Proposition $8(E, \mathcal{L})$ satisfies the Euler formula for zero sets. This proves the implication (i) $\Rightarrow$ (iii).

As for $($ iii $) \Rightarrow\left(\right.$ ii), we proceed by induction on $\# X^{0}$ for $X \in \mathcal{L}$. Assume that $X^{0}$ is not empty. Pick $e \in X^{0}$ and delete the coordinate subset $X^{0} \backslash e$ from $X$. Consider the topal fiber $\mathcal{R}=\left\{X^{\prime} \in \mathcal{L}: X^{\prime} \backslash \underline{X}=X \backslash \underline{X}\right\}$ relative to $X$ and $\underline{X}$, and contract $\mathcal{R}$ to $\mathcal{R} /\left(X^{0} \backslash e\right)$. Let $U^{(e)}$ denote the (unit) sign vector on $E$ with $U_{e}^{(e)}=+1$ and $U_{f}^{(e)}=0$ for $f \neq e$. Since $\mathcal{R} /\left(X^{0} \backslash e\right)$ satisfies the Euler-Poincaré formula for zero sets, both $X \circ U^{(e)}$ and $X \circ-U^{(e)}$ must belong to $\mathcal{L}$. By the induction hypothesis

$$
\left(X \circ U^{(e)}\right) \circ Z,\left(X \circ-U^{(e)}\right) \circ Z \in \mathcal{L} \text { for all } Z \in\{ \pm 1\}^{E},
$$

whence indeed $X \circ Y \in \mathcal{L}$ for all $Y \in\{ \pm 1\}^{E}$. 
To prove the final implication (ii) $\Rightarrow(\mathrm{i})$, we employ the recursive characterization of Theorem 3. Since (IC) holds by the implication for $X \in \mathcal{L}$ in (ii), property (1) of this theorem is trivially fulfilled. Observe that $\{ \pm 1\} \subseteq\left\{X_{e}: X \in \mathcal{L}\right\}$ because the topal fiber relative to $X \in \mathcal{L}$ and $\underline{X} \neq E$ contains all possible $-1,+1$ entries. If $X, Y$ are two topes of $\mathcal{L}$ with $S(X, Y)=\{e\}$, then the topal fiber relative to $X$ and $E \backslash e$ must contain $\frac{1}{2}(X+Y)$ by virtue of the Euler-Poincaré formula for zero sets. This establishes property (4) and (RN1).

Suppose that the topes of $\mathcal{L}$ do not form a partial cube in $\{ \pm 1\}^{E}$. Then choose topes $X$ and $Y$ with $\# S(X, Y) \geq 2$ as small as possible such that the topal fiber $\mathcal{R}$ relative to $X$ and $E \backslash S(X, Y)$ include no other topes than $X$ or $Y$. The formula for zero sets implies that this topal fiber $\mathcal{R}$ must contain at least some $Z \in \mathcal{L}$ with $Z^{0}$ of odd cardinality. Then for $e \neq f$ in $S(X, Y)$ one can select signs for some tope $Z^{\prime}$ conforming to $Z$ such that $Z_{e}^{\prime} Z_{f}^{\prime} \neq X_{e} X_{f}=Y_{e} Y_{f}$. Hence $\mathcal{R}$ contains the tope $Z^{\prime}$ that is different from $X$ and $Y$, contrary to the hypothesis. This contradiction establishes that $\mathcal{L}$ fulfills property (3) and is semisimple.

Consider the hyperplane $\mathcal{L}_{e}^{0}$ and the corresponding halfspaces $\mathcal{L}_{e}^{+}$and $\mathcal{L}_{e}^{-}$( which are two disjoint topal fibers of $\mathcal{L}$ ). Then the formula

$$
\sum_{X \in \mathcal{L}}(-1)^{\# X^{0}}-\sum_{Y \in \mathcal{L}_{e}^{+}}(-1)^{\# Y^{0}}-\sum_{Z \in \mathcal{L}_{e}^{-}}(-1)^{\# Z^{0}}=-1
$$

amounts to

$$
\sum_{W \in \mathcal{L}_{e}^{0} \backslash e}(-1)^{\# W^{0}}=1
$$

showing that the hyperplane after semisimplification satisfies the Euler-Poincare formula. The analogous conclusion holds for any topal fiber $\mathcal{L} \backslash A$ of any $X \in \mathcal{L}$ with $A \subseteq \underline{X}$ because taking topal fibers and contractions commute. By induction we conclude that $(E, \mathcal{L})$ is a COM satisfying (IC), that is, a lopsided system.

Note that the equivalence of (i) and (ii) in Proposition 9 rephrases a result by Wiedemann [38] on lopsided sets. Observe that in condition (iii) one cannot dispense with contractions as the example $\mathcal{L}=\{+00\}$ shows. Neither can one weaken condition (ii) by dismissing topal fibers: consider a path in the 1 -skeleton of $[-1,+1]^{3}$ connecting five vertices of the solid cube, which would yield an induced but non-isometric path of the corresponding graphical 3 -cube. Let $\mathcal{L}$ comprise the five vertices and the barycenters of the four edges, being represented by their sign vectors. Then all topal fibers except one satisfy the first statement in (ii), the second one being trivially satisfied.

\section{RANKING COMs}

Particular COMs naturally arise in order theory. For the entire section, let $(P, \leq)$ denote an ordered set (alias poset), that is, a finite set $P$ endowed with an order (relation) $\leq$. A ranking (alias weak order) is an order for which incomparability is transitive. Equivalently, an order $\leq$ on $P$ is a ranking exactly when $P$ can be partitioned into antichains (where an antichain is a set of mutually incomparable elements) $A_{1}, \ldots, A_{k}$, such that $x \in A_{i}$ is below 
$y \in A_{j}$ whenever $i<j$. An order $\leq$ on $P$ is linear if any two elements of $P$ are comparable, that is, all antichains are trivial (i.e., of size $<2$ ). An order $\leq^{\prime}$ extends an order $\leq$ on $P$ if $x \leq y$ implies $x \leq^{\prime} y$ for all $x, y \in P$. Of particular interest are the linear extensions and, more generally, the ranking extensions of a given order $\leq$ on $P$.

Let us now see how to associate a set of sign vectors to an order $\leq$ on $P=\{1,2, \ldots, n\}$. For this purpose take $E$ to be the set of all 2-subsets of $P$ and encode $\leq$ by its characteristic sign vector $X^{\leq} \in\{0, \pm 1\}^{E}$, which to each 2-subset $e=\{i, j\}$ assigns $X_{e}^{\leq}=0$ if $i$ and $j$ are incomparable, $X_{e}^{\leq}=+1$ if the order agrees with the natural order on the 2 -subset $e$, and else $X_{e}^{\leq}=-1$. In the sign vector representation the different components are ordered with respect to the lexicographic natural order of the 2-subsets of $P$.

The composition of sign vectors from different orders $\leq$ and $\leq^{\prime}$ does not necessarily return an order again. Take for instance, $X^{\leq}=+++$coming from the natural order on $P$ and $X^{\leq^{\prime}}=0-0$ coming from the order with the single (nontrivial) comparability $3 \leq^{\prime} 1$. The composition $X \leq^{\prime} \circ X^{\leq}$equals +-+ , which signifies a directed 3 -cycle and thus no order. The obstacle here is that $X^{\leq}$encodes an order for which one element is incomparable with a pair of comparable elements. Transitivity of the incomparability relation is therefore a necessary condition for obtaining a COM.

We denote by $\mathcal{R}(P, \leq)$ the simplification of the set of sign vectors associated to all ranking extensions of $(P, \leq)$. Note that the simplification amounts to omitting the pairs of the ground set corresponding to pairs of comparable pairs of $P$.

Theorem 4. Let $(P, \leq)$ be an ordered set. Then $\mathcal{R}(P, \leq)$ is a realizable COM, called the ranking $\mathrm{COM}$ of $(P, \leq)$.

Proof. The composition $X \circ Y$ of two sign vectors $X$ and $Y$ which encode rankings has an immediate order-theoretic interpretation: each (maximal) antichain of the order $\leq_{X}$ encoded by $X$ gets ordered according to the order $\leq_{Y}$ corresponding to $Y$. Similarly, in order to realize $X \circ-Y$ one only needs to reverse the order $\leq_{Y}$ before imposing it on the antichains of $\leq_{X}$. This establishes conditions (C) and (FS). To verify strong elimination ( $\mathrm{SE}^{=}$), assume that $X$ and $Y$ are given with $\underline{X}=\underline{Y}$, so that the corresponding rankings have the same antichains. These antichains may therefore be contracted (and at the end of the process get restored again). Now, for convenience we may assume that $X$ is the constant +1 vector, thus representing the natural linear order on $P$. Given $e=\{i, j\}$ with $i<_{X} j$, let $Y_{e}=$ -1 , that is, $j<_{Y} i$. To construct a sign vector $Z$ with $Z_{e}=0$ and $Z_{f}=X_{f}$ whenever $Y_{f}=X_{f}$, take the sign vector of the ranking corresponding to $X$ but place the subchain $\left\{h: i<_{X} h<_{X} j\right.$ and $\left.h<_{Y} j\right\}$ directly below the newly created antichain $\{i, j\}$, and $\left\{h: i<_{X} h<_{X} j\right.$ and $\left.j<_{Y} h\right\}$ directly above it, while leaving everything else in the natural order. This establishes that $\mathcal{R}(P, \leq)$ is a COM. Realizability of $\mathcal{R}(P, \leq)$ will be confirmed in the third paragraph below.

To provide an example for a ranking COM and also illustrate the preceding construction, consider the ordered set ("fence") shown in Figure 4(a), In Figure 4(b), the sign vector $X$ 
encodes the natural order $<$ and $Y$ the ordering $3<_{Y} 2<_{Y} j=5<_{Y} i=1<_{Y} 4$, while $Z$ encodes the intermediate ranking with $2<_{Z} 3<_{Z} 1$ and $5<_{Z} 4$.

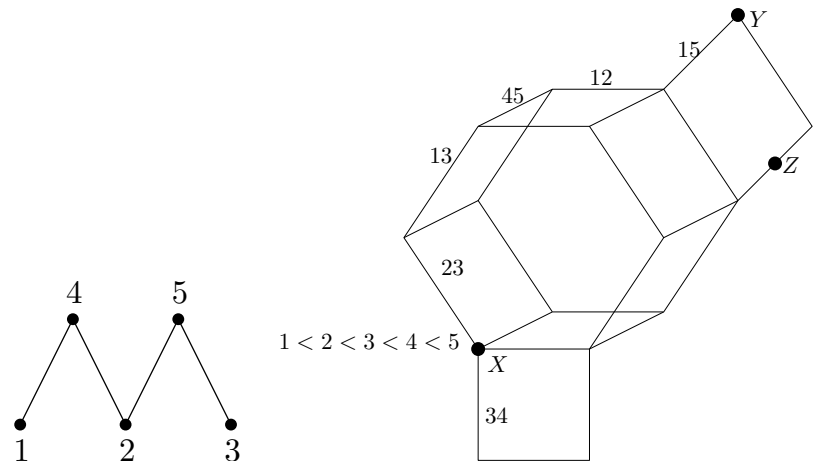

(a)

(b)

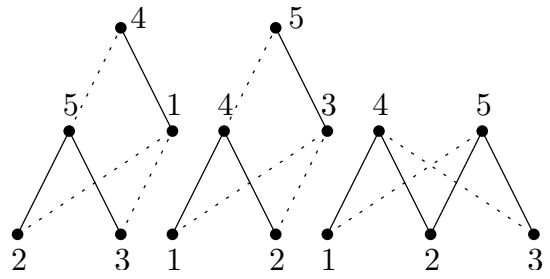

(c)

Figure 4. From (a) an ordered set $(P, \leq)$ to (b) the ranking $\operatorname{COM} \mathcal{R}(P, \leq)$ comprising three maximal faces determined by (c) the minimal rankings in $\mathcal{R}(P, \leq)$.

The ranking $\operatorname{COM} \mathcal{R}(P, \leq)$ is the natural host of all linear extensions of $(P, \leq)$ (as its topes), where the interconnecting rankings signify the cell structure. The linear extension graph of an ordered set $(P, \leq)$ is defined on the linear extensions of $(P, \leq)$, where two linear extensions are joined by an edge iff they differ on the order of exactly one pair of elements. Thus, the linear extension graph of $(P, \leq)$ is the tope graph of $\mathcal{R}(P, \leq)$. A number of geometric and graph-theoretical features of linear extensions have been studied by various authors $33,35,36$, which can be expressed most naturally in the language of COMs.

One such result translates to the fact that ranking COMs are realizable. To see this, first consider the braid arrangement of type $B_{n}$, i.e., the central hyperplane arrangement $\left\{H_{i j}: 1 \leq i<j \leq n\right\}$ in $\mathbb{R}^{n}$, where $H_{i j}=\left\{x \in \mathbb{R}^{n}: x_{i}=x_{j}\right\}$ and the position of any point in the corresponding halfspace $\left\{x \in \mathbb{R}^{n}: x_{i}<x_{j}\right\}$ is encoded by + with respect to $H_{i j}$. The resulting $\mathrm{OM}$ is known as the permutahedron [10]. Given an order $\unlhd$ on $P=$ $\{1, \ldots, n\}$ consider the arrangement $E=\left\{H_{i j}: i, j\right.$ incomparable $\}$ restricted to the open polyhedron $\bigcap_{i \triangleleft j}\left\{x \in \mathbb{R}^{n}: x_{i}<x_{j}\right\}$. The closure of the latter intersected with the unit cube $[0,1]^{n}$ coincides with the order polytope [37] of $(P, \unlhd)$. It is well-known that the maximal cells of the braid arrangement restricted to the order polytope of $(P, \unlhd)$ correspond to the linear extensions of $(P, \unlhd)$. Thus, the COM realized by the order polytope and the braid arrangement has the same set of topes as the ranking COM. By the results of Section 4, this implies that both COMs coincide. In particular, ranking COMs are realizable.

We will now show how other notions for general COMs translate to ranking COMs. A face $\mathcal{F}$ of $\mathcal{R}(P, \leq)$, as defined in Section 3, can be viewed as the set of all rankings that extend some ranking extension $\leq^{\prime}$ of $\leq$. Hence $\mathcal{F} \cong \mathcal{R}\left(P, \leq^{\prime}\right)$, i.e., all faces of a ranking COM are also 
ranking COMs. The minimal elements of $\mathcal{R}(P, \leq)$ with respect to sign ordering (being the improper cocircuits of $\mathcal{R}(P, \leq))$ are the minimal ranking extensions of $(P, \leq)$, see Figure 4(c). A hyperplane $\mathcal{R}_{e}^{0}$ of $\mathcal{R}:=\mathcal{R}(P, \leq)$ relative to $e=\{i, j\} \in E$ corresponds to those ranking extensions of $(P, \leq)$ leaving $i, j$ incomparable. Thus, $\mathcal{R}_{e}^{0}$ can be seen as the ranking COM of the ordered set obtained from $(P, \leq)$ by identifying $i$ and $j$. The open halfspace $\mathcal{R}_{e}^{+}$ corresponds to those ranking extensions fixing the natural order on $i, j$ and is therefore the ranking COM of the ordered set $(P, \leq)$ extended with the natural order on $i, j$. The analogous statement holds for $\mathcal{R}_{e}^{-}$. Similarly, the carrier of $\mathcal{R}$ relative to $e$ can be seen as the ranking $\mathrm{COM}$ of the ordered set arising as the intersection of all minimal rankings of $(P, \leq)$ not fixing an order on $i, j$. So, in all three cases the resulting COMs are again ranking COMs.

One may wonder which are the ordered sets whose ranking COM is an OM or a lopsided system. The maximal cells in Figure 4(b) are symmetric and therefore correspond to OMs.

Proposition 10. The ranking COM of $(P, \leq)$ is an $O M$ if and only if $\leq$ is a ranking. In this case, $\mathcal{R}(P, \leq)$ and its proper faces are products of permutohedra.

Proof. Since any $\mathrm{OM}$ has a unique improper cocircuit, $\leq$ needs to be a ranking in order to have that $\mathcal{R}(P, \leq)$ is an OM. On the other hand, it is easy to see that if $\leq$ is a ranking on $P$, then $\mathcal{R}(P, \leq)$ is a product of permutohedra and, in particular, is symmetric, yielding the claim.

Proposition 11. The ranking COM of $(P, \leq)$ is a lopsided system if and only if $(P, \leq)$ has width at most 2. In this case, the tope graph of $\mathcal{R}(P, \leq)$ is the covering graph (i.e., undirected Hasse diagram) of a distributive lattice.

Proof. If $(P, \leq)$ contains an antichain of size 3 , then the corresponding face of $\mathcal{R}(P, \leq)$ does not satisfy ideal composition, so that $\mathcal{R}(P, \leq)$ is not lopsided. Conversely, if all antichains have size at most 2 , then the zero entries of a sign vector $X$ encoding a ranking of $(P, \leq$ ) correspond to maximal antichains of size 2. Thus, choosing any sign on a zero entry just corresponds to fixing a linear order on the two elements of the antichain. Since these antichains are maximal and $X$ encodes a ranking, the resulting sign vector encodes a ranking, too. This proves ideal composition.

Let us now prove the second part of the claim. By Dilworth's Theorem [17], $(P, \leq)$ can be covered by two disjoint chains, $C$ and $D$. A linear extension of $(P, \leq)$ corresponds to an order-preserving mapping of $C$ to positions between consecutive elements of $D$ or above or below its maximal or minimal element, respectively. A linear extension $\unlhd$ of $(P, \leq)$ can thus be codified by an order-preserving mapping $f$ from $C$ to the chain $\hat{D}=D \cup \hat{1}$, i.e., $D$ with a new top element $\hat{1}$ added: $f(c)=d \in \hat{D}$ signifies that the subchain $f^{-1}(d)$ of $C$ immediately precedes $d$ in the linear extension $\unlhd$. If there are no comparabilities between $C$ and $D$ in $(P, \leq)$, then the tope graph of $\mathcal{R}(P, \leq)$ is the covering graph of the entire distributive lattice $L$ of order-preserving mappings from $C$ to $\hat{D}$ since covering pairs in $L$ correspond to pairs of linear extensions which are distinguished by a single neighbors swap. Additional covering relations between $C$ and $D$ yield lower and upper bounds for the order-preserving mappings, 
whence the resulting (distributive) linear-extension lattice of $(P, \leq)$ constitutes some orderinterval of $L$.

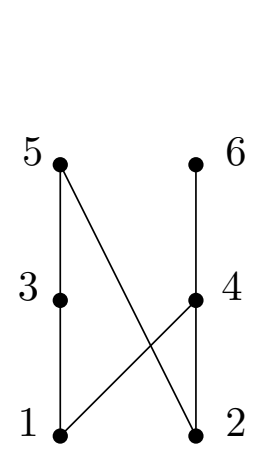

(a)

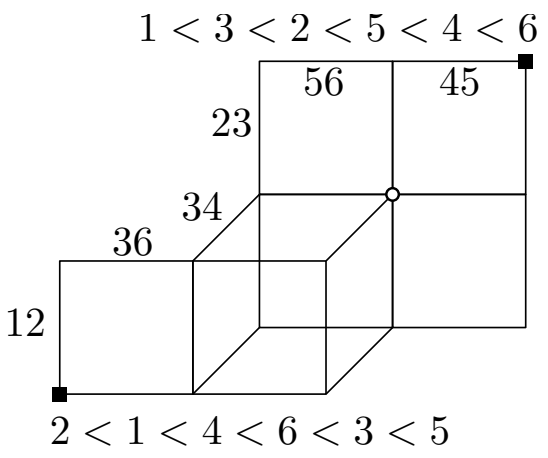

(b)

Figure 5. From (a) the Hasse diagram of $(P, \leq)$ having width 2 to (b) the tope graph of the lopsided system $\mathcal{R}(P, \leq)$ oriented as a distributive lattice.

In Figure 5(a), an ordered set $(P, \leq)$ of width 2 is displayed, which has the natural order on $\{1, \ldots, 6\}$ among its linear extensions. Figure $5(\mathrm{~b})$ shows the tope graph of the lopsided system $\mathcal{R}(P, \leq)$ and highlights the pair of diametrical vertices that determine the distributive lattice orientation (and its opposite); the natural order is associated with the (median) vertex (indicated by a small open circle). If we added the compatibility $3<6$ to the Hasse diagram, then the tope graph shrinks by collapsing the (two) edges corresponding to $\{3,6\}$. The resulting graph with $F_{7}=13$ vertices is known as the "Fibonacci cube of order 5".

More generally, the Fibonacci cube of order $n-1 \geq 1$ is canonically obtained as the tope graph of $\mathcal{R}(\{1, \ldots n\}, \leq)$, where $(\{1, \ldots n\}, \leq)$ is the ordered set determined by the (cover) comparabilities $1<3<5<\ldots<2\left\lfloor\frac{n-1}{2}\right\rfloor+1$ and $2<4<6<\ldots<2\left\lfloor\frac{n}{2}\right\rfloor$ and $k<k+3$ for all $k=1, \ldots, n-3$. The incomparable pairs thus form the set $E=\{\{i, i+1\}: i=1, \ldots, n-1\}\}$. The intersection graph of $E$ is a path of length $n-1$, which yields a "fence" when orienting its edges in a zig-zag fashion. This fence and its opposite yield the mutually opposite ordered sets of supremum-irreducibles for the (distributive) "Fibonacci" lattice and its opposite. Recall that the opposite $(R, \leq)^{o p}=\left(R, \leq^{o p}\right)$ of an ordered set $(R, \leq)$ is defined by switching $\leq$ to $\geq$, that is: $x \leq^{o p} y$ if and only if $y \leq x$.

Similarly, to the fact that hyperplanes, carriers, and open halfspaces of a ranking COM are also ranking COMs, the class of ranking COMs is also closed with respect to contractions. On the other hand deleting an element in a ranking COM may give a COM which is not a ranking COM.

To give a small example, consider the minor $\mathcal{R}(P, \leq) \backslash\{5,6\}$ of the lopsided system $\mathcal{R}(P, \leq)$ of Figure 5. Suppose by way of contradiction, that $\mathcal{R}(P, \leq) \backslash\{5,6\}$ could be represented by some $\mathcal{R}(Q, \leq)$. The ordered set $(Q, \leq)$ must be of width 2 since the tope graph of $\mathcal{R}(Q, \leq)$ is obtained from the graph in Figure 5(b) by contracting the five edges labeled 56 and thus 
includes no 3-cube. We can keep the current labeling without loss of generality to the point that $Q$ must include four antichains $\{1,2\},\{2,3\},\{3,4\},\{3,6\}$ of size 2 with exactly this intersection pattern. But then the fifth antichain must be disjoint from $\{1,2\}$ and $\{2,3\}$ but intersecting both $\{3,4\}$ and $\{3,6\}$, whence it must be $\{4,6\}$, which however yields a contradiction as $\{3,4,6\}$ cannot be an antichain in $(Q, \leq)$. Furthermore, $\mathcal{R}(P, \leq) \backslash\{5,6\}$ is easily seen to be the COM amalgam of ranking COMs, i.e., the class is also not closed under COM amalgamations.

It would be interesting to determine the smallest minor-closed class of COMs containing the ranking COMs.

\section{COMs AS COMPleXes OF ORIENTED MATROIDS}

In this section we consider a topological approach to COMs. In the subsequent definitions, notations, and results we closely follow Section 4 of [10] (some missing definitions can be also found there). Let $B^{d}=\left\{x \in \mathbb{R}^{d}:\|x\| \leq 1\right\}$ be the standard $d$-ball and its boundary $S^{d-1}=\partial B^{d}=\left\{x \in \mathbb{R}^{d}:\|x\|=1\right\}$ be the standard $(d-1)$-sphere. When saying that a topological space $T$ is a "ball" or a "sphere", it is meant that $T$ is homeomorphic to $B^{d}$ or $S^{d-1}$ for some $d$, respectively.

11.1. Regular cell complexes. A (regular) cell complex $\Delta$ constitutes of a covering of a Hausdorff space $\|\Delta\|=\bigcup_{\sigma \in \Delta} \sigma$ with finitely many subspaces $\sigma$ homeomorphic with (open) balls such that (i) the interiors of $\sigma \in \Delta$ partition $\|\Delta\|$ (i.e., every $x \in\|\Delta\|$ lies in the interior of a single $\sigma \in \Delta$ ), (ii) the boundary $\partial \sigma$ of each ball $\sigma \in \Delta$ is a union of some members of $\Delta$ [10, Definition 4.7.4]. Additionally, we will assume that $\Delta$ obeys the intersection property (iii) whenever $\sigma, \tau \in \Delta$ have non-empty intersection then $\sigma \cap \tau \in \Delta$. The balls $\sigma \in \Delta$ are called cells of $\Delta$ and the space $\|\Delta\|$ is called the underlying space of $\Delta$. If $T$ is homeomorphic to $\|\Delta\|$ (notation $T \cong\|\Delta\|$ ), then $\Delta$ is said to provide a regular cell decomposition of the space $T$. We will say that a regular cell complex $\Delta$ is contractible if the topological space $\|\Delta\|$ is contractible. If $\sigma, \tau \in \Delta$ and $\tau \subseteq \sigma$, then $\tau$ is said to be a face of $\sigma . \Delta^{\prime} \subseteq \Delta$ is a subcomplex of $\Delta$ if $\tau \in \Delta^{\prime}$ implies that every face of $\tau$ also belongs to $\Delta^{\prime}$. The 0 -cells and 1-cells of $\Delta$ are called vertices and edges. The 1-skeleton of $\Delta$ is encoded by the graph $G(\Delta)$ consisting of the vertices of $\Delta$ and graph edges corresponding to the edges of $\Delta$. The set of cells of $\Delta$ ordered by containment is denoted by $\mathcal{F}(\Delta)$ (in $[13], \mathcal{F}(\Delta)$ is also called an abstract cell complex). Two cell complexes $\Delta$ and $\Delta^{\prime}$ are combinatorially equivalent if their ordered sets $\mathcal{F}(\Delta)$ and $\mathcal{F}\left(\Delta^{\prime}\right)$ are isomorphic. We continue by recalling several results relating regular cell complexes.

The order complex of a finite ordered set $P$ is an abstract simplicial complex $\Delta_{\text {ord }}(P)$ whose vertices are the elements of $P$ and whose simplices are the chains $x_{0}<x_{1}<\cdots<x_{k}$ of $P$. The geometric realization $\|\Delta\|$ of a complex $\Delta$ basically consists of simultaneously replacing all abstract simplices by geometric simplices, see $[12$ for a formal definition. In particular, $\left\|\Delta_{\text {ord }}(P)\right\|$ is the geometric realization of $\Delta_{\text {ord }}(P)$. For an element $x$ of $P$ let 
$P_{<x}=\{y \in P: y<x\}$ and $P_{\leq x}=P_{<x} \cup\{x\}$. The following fact expresses that a regular cell complex is homeomorphic to the order complex of its ordered set of faces.

Proposition 12. [10, Proposition 4.7.8] Let $\Delta$ be a regular cell complex. Then $\|\Delta\| \cong$ $\| \Delta_{\text {ord }}(\mathcal{F}(\Delta) \|$. Moreover, this homeomorphism can be chosen to be cellular, i.e., it restricts to a homeomorphism between $\sigma$ and $\left\|\Delta_{\text {ord }}\left(\mathcal{F}_{\leq \sigma}\right)\right\|$, for all $\sigma \in \Delta$.

The ordered sets of faces of regular cell complexes can be characterized in the following way:

Proposition 13. [10, Proposition 4.7.23] Let $P$ be an ordered set. Then $P \cong \mathcal{F}(\Delta)$ for some regular cell complex $\Delta$ if and only if $\left\|\Delta_{\text {ord }}\left(P_{<x}\right)\right\|$ is homeomorphic to a sphere for all $x \in P$. Furthermore, $\Delta$ is uniquely determined by $P$ up to a cellular homeomorphism.

11.2. Cell complexes of OMs. Now, let $\mathcal{L} \subseteq\{ \pm 1,0\}^{E}$ be the set of covectors of an oriented matroid. Then $(\mathcal{L}, \leq)$ is a semilattice with least element $\mathbf{0}$ (where $\leq$ is the product ordering on $\{ \pm 1,0\}^{E}$ defined above). The semilattice $(\mathcal{L} \cup\{\hat{1}\}, \leq)$, i.e., the semilattice $\mathcal{L}$ with a largest element $\hat{1}$ adjoined, is a lattice, called the big face lattice of $\mathcal{L}$ and denoted by $\mathcal{F}_{\text {big }}(\mathcal{L})$. Let $\mathcal{F}_{\text {big }}(\mathcal{L})^{o p}$ denote the opposite of $\mathcal{F}_{\text {big }}(\mathcal{L})$.

Proposition 14. [10, Corollary 4.3.4 \& Lemma 4.4.1] Let $(E, \mathcal{L})$ be an oriented matroid of rank $r$. Then $\mathcal{F}_{\text {big }}(\mathcal{L})^{o p}$ is isomorphic to the face lattice of a $P L$ (Piecewise Linear) regular cell decomposition of the $(r-1)$-sphere, denoted by $\Delta(\mathcal{L})$. The tope graph of $\mathcal{L}$ encodes the 1-skeleton of $\Delta(\mathcal{L})$.

11.3. Cell complexes of COMs. We collected all ingredients necessary to associate to each COM a regular cell complex. Let $\mathcal{L} \subseteq\{ \pm 1,0\}^{E}$ be the set of covectors of a COM. Analogously to oriented matroids, let $\mathcal{F}_{\text {big }}(\mathcal{L}):=(\mathcal{L} \cup\{\hat{1}\}, \leq)$ denote the ordered set $\mathcal{L}$ with a top element $\hat{1}$ adjoined and call $\mathcal{F}_{\text {big }}(\mathcal{L})$ the big face semilattice of $\mathcal{L}$. Let $\mathcal{F}_{\text {big }}(\mathcal{L})^{\text {op }}$ denote the opposite of $\mathcal{F}_{b i g}(\mathcal{L}) . \mathcal{F}_{b i g}(\mathcal{L})^{o p}$ is isomorphic to the semilattice comprising the empty set and the faces of $\mathcal{L}$ ordered by inclusion. Recall that for any $X \in \mathcal{L}$, the deletion $(E \backslash \underline{X}, F(X) \backslash \underline{X})$ corresponding to the face $F(X)$ is an oriented matroid, which we will denote by $\mathcal{L}(X)$. Since $F(Y) \subseteq F(X)$ if and only if $Y \in F(X)$, the order ideal $\mathcal{F}_{b i g}(\mathcal{L})_{\leq X}^{o p}$ coincides with the interval $[\hat{1}, X]$ of $\mathcal{F}_{\text {big }}(\mathcal{L})^{o p}$ and is isomorphic to the opposite big face lattice $\mathcal{F}_{\text {big }}(\mathcal{L}(X))^{\text {op }}$ of $\mathcal{L}(X)$. By Proposition 14, if $r$ is the rank of $\mathcal{L}(X)$, then $\mathcal{F}_{b i g}(\mathcal{L}(X))^{\text {op }}$ is isomorphic to the face lattice of a PL cell decomposition $\Delta(\mathcal{L}(X))$ of the $(r-1)$-sphere. Additionally, the tope graph of $\mathcal{L}(X)$ encodes the 1-skeleton of $\Delta(\mathcal{L}(X))$. Denote by $\sigma(\mathcal{L}(X))$ the open PL ball whose boundary is the $(r-1)$-sphere occurring in the definition of $\Delta(\mathcal{L}(X))$. We will call the cells of $\Delta(\mathcal{L}(X))$ faces of $\sigma(\mathcal{L}(X))$. The faces of $\Delta(\mathcal{L}(X))$ correspond to the elements of $\mathcal{L}(X) \cup\{\hat{1}\}$. Notice in particular that the adjoined element $\hat{1}$ corresponds to the empty face in $\Delta(\mathcal{L}(X))$ and $\mathbf{0} \in F(X) \backslash \underline{X}$ corresponds to the unique maximal face $\sigma(\mathcal{L}(X))$.

By Proposition 12, for any $X \in \mathcal{L}$ we have $\|\Delta(\mathcal{L}(X))\| \cong\left\|\Delta_{\text {ord }}\left(\mathcal{F}_{\text {big }}(\mathcal{L}(X))^{o p}\right)\right\|$. Furthermore, since $\mathcal{F}_{\text {big }}(\mathcal{L}(X))^{o p}$ is isomorphic to $\mathcal{F}_{\text {big }}(\mathcal{L})_{\leq X}^{o p},\left\|\Delta_{\text {ord }}\left(\mathcal{F}_{\text {big }}(\mathcal{L}(X))^{o p}\right)\right\| \cong$ $\left\|\Delta_{\text {ord }}\left(\mathcal{F}_{\text {big }}(\mathcal{L})_{\leq X}^{o p}\right)\right\|$. Thus for each $X \in \mathcal{L},\left\|\Delta_{\text {ord }}\left(\mathcal{F}_{\text {big }}(\mathcal{L})_{<X}^{o p}\right)\right\|$ is homeomorphic to 
$\|\Delta(\mathcal{L}(X)) \backslash \sigma(\mathcal{L}(X))\|$, which is a sphere by Proposition 14 . Now, by Proposition $13, \mathcal{F}_{\text {big }}(\mathcal{L})^{\text {op }}$ is the face semilattice of a regular cell complex $\Delta(\mathcal{L})$. Moreover, from the proof of Proposition 13 it follows that $\Delta(\mathcal{L})$ can be chosen so that its cells are the balls $\sigma(\mathcal{L}(X)), X \in \mathcal{L}$, whose boundary spheres are decomposed by $\Delta(\mathcal{L}(X))$. Since $F(X) \cap F(Y)=F(X \circ Y)$ for any two covectors $X, Y \in \mathcal{L}$ such that $F(X)$ and $F(Y)$ intersect, $\mathcal{F}_{b i g}(\mathcal{L}(X \circ Y))^{o p}$ is isomorphic to a sublattice of $\mathcal{F}_{\text {big }}(\mathcal{L}(X))^{o p}$ and to a sublattice of $\mathcal{F}_{\text {big }}(\mathcal{L}(Y))^{o p}$. Therefore the cells $\Delta(\mathcal{L}(X))$ and $\Delta(\mathcal{L}(Y))$ are glued in $\Delta(\mathcal{L})$ along $\Delta(\mathcal{L}(X \circ Y))$, whence $\Delta(\mathcal{L})$ also satisfies the intersection property (iii). Notice also that since the 1-skeleton of each $\Delta(\mathcal{L}(X))$ yields the tope graph of $\mathcal{L}(X)$ and $\Delta(\mathcal{L})$ satisfies (iii), the 1-skeleton of $\Delta(\mathcal{L})$ encodes the tope graph of $\mathcal{L}$. We summarize this in the following proposition, in which we also establish that $\Delta(\mathcal{L})$ is contractible.

Proposition 15. If $(E, \mathcal{L})$ is a COM, then $\Delta(\mathcal{L})$ is a contractible regular cell complex and the tope graph of $\mathcal{L}$ is realized by the 1-skeleton of $\Delta(\mathcal{L})$.

Proof. We prove the contractibility of $\Delta(\mathcal{L})$ by induction on the number of maximal cells of $\Delta(\mathcal{L})$ by using the so-called gluing lemma $[7$, Lemma 10.3] and our decomposition procedure (Proposition 7) for COMs. By the gluing lemma, if $\Delta$ is a cell complex which is the union of two contractible cell complexes $\Delta^{\prime}$ and $\Delta^{\prime \prime}$ such that their intersection $\Delta_{0}=\Delta^{\prime} \cap \Delta^{\prime \prime}$ is contractible, then $\Delta$ is contractible.

If $\Delta(\mathcal{L})$ consists of a single maximal cell $\sigma(\mathcal{L}(X))$, then $(E, \mathcal{L})$ is an OM and therefore is contractible. Otherwise, as shown above there exists an element $e \in E$ such that if we set $\mathcal{L}^{\prime}:=\mathcal{L}_{e}^{-}$and $\mathcal{L}^{\prime \prime}:=\overline{\mathcal{L}_{e}^{+}}$, then $(E, \mathcal{L})$ is the COM amalgam of the COMs $\left(E, \mathcal{L}^{\prime}\right)$ and $\left(E, \mathcal{L}^{\prime \prime}\right)$ along the $\mathrm{COM} \mathcal{L}^{\prime} \cap \mathcal{L}^{\prime \prime}=N^{-}\left(\mathcal{L}_{e}^{0}\right)$. By induction hypothesis, the cell complexes $\Delta\left(\mathcal{L}^{\prime}\right), \Delta\left(\mathcal{L}^{\prime \prime}\right)$, and $\Delta\left(\mathcal{L}^{\prime} \cap \mathcal{L}^{\prime \prime}\right)$ are contractible. Each maximal cell of $\Delta(\mathcal{L})$ corresponds to a maximal face of $\mathcal{L}$, thus by Proposition 7 it is a maximal cell of $\Delta\left(\mathcal{L}^{\prime}\right)$, of $\Delta\left(\mathcal{L}^{\prime \prime}\right)$, or of both (in which case it belongs to $\left.\Delta\left(\mathcal{L}^{\prime} \cap \mathcal{L}^{\prime \prime}\right)\right)$. Since each cell of $\Delta(\mathcal{L})$ belongs to a maximal cell, this implies that $\Delta(\mathcal{L}) \subseteq \Delta\left(\mathcal{L}^{\prime}\right) \cup \Delta\left(\mathcal{L}^{\prime \prime}\right)$. Since $\mathcal{L}^{\prime} \cup \mathcal{L}^{\prime \prime} \subseteq \mathcal{L}$, we also have the converse inclusion $\Delta\left(\mathcal{L}^{\prime}\right) \cup \Delta\left(\mathcal{L}^{\prime \prime}\right) \subseteq \Delta(\mathcal{L})$. Finally, since $\mathcal{L}^{\prime} \cap \mathcal{L}^{\prime \prime}=N^{-}\left(\mathcal{L}_{e}^{0}\right)$, the definition of carriers implies that $\Delta\left(\mathcal{L}^{\prime} \cap \mathcal{L}^{\prime \prime}\right)=\Delta\left(\mathcal{L}^{\prime}\right) \cap \Delta\left(\mathcal{L}^{\prime \prime}\right)$.

11.4. Zonotopal COMs. As in the introduction, let $E$ be a central arrangement of $n$ hyperplanes of $\mathbb{R}^{d}$ and $\mathcal{L}$ be the oriented matroid corresponding to the regions of $\mathbb{R}^{d}$ defined by this arrangement. Let $\mathbf{X}=\left\{\mathbf{x}_{1}, \ldots, \mathbf{x}_{n}\right\}$ be a set of unit vectors each normal to a different hyperplane of $E$. The zonotope $\mathcal{Z}:=\mathcal{Z}(\mathbf{X})$ of $\mathbf{X}$ is the convex polytope of $\mathbb{R}^{d}$ which can be expressed as the Minkowski sum of $n$ line segments

$$
\mathcal{Z}=\left[-\mathbf{x}_{1}, \mathbf{x}_{1}\right]+\left[-\mathbf{x}_{2}, \mathbf{x}_{2}\right]+\ldots+\left[-\mathbf{x}_{n}, \mathbf{x}_{n}\right] .
$$

Equivalently, $\mathcal{Z}$ is the projection of the $n$-cube $C_{n}:=\left\{\sum_{i=1}^{n} \lambda_{i} \mathbf{e}_{i}:-1 \leq \lambda_{i} \leq+1\right\} \subset \mathbb{R}^{n}$ under $\mathbf{X}$ (where $\mathbf{e}_{1}, \ldots, \mathbf{e}_{n}$ denotes the standard basis of $\mathbb{R}^{n}$ ), which sends $\mathbf{e}_{i}$ to $\mathbf{x}_{i}, i=1, \ldots, n$ :

$$
\mathcal{Z}=\left\{\sum_{i=1}^{n} \lambda_{i} \mathbf{x}_{i}:-1 \leq \lambda_{i} \leq+1\right\} \subset \mathbb{R}^{d} .
$$


The hyperplane arrangement $E$ is geometrically polar to $\mathcal{Z}$ : the regions of the arrangement are the cones of outer normals at the faces of $\mathcal{Z}$. The face lattice of $\mathcal{Z}$ is opposite (antiisomorphic) to the big face lattice of the oriented matroid $\mathcal{L}$ of $\mathbf{X}$, that is, $\mathcal{F}(\mathcal{Z}) \simeq \mathcal{F}_{\text {big }}(\mathcal{L})^{o p}$; for this and other results, see [10, Subsection 2.2]. Therefore the zonotopes together with their faces can be viewed as the cell complexes associated to realizable oriented matroids.

The following properties and examples of zonotopes are well-known:

- any face of a zonotope is a zonotope;

- a polytope $P$ is a zonotope if and only if every 2-dimensional face of $P$ is a zonotope and if and only if every 2-dimensional face of $P$ is centrally symmetric;

- two zonotopes are combinatorially equivalent if and only if their 1-skeletons yield isomorphic graphs;

- the $d$-cube is the zonotope corresponding to the arrangement of coordinate hyperplanes (called also Boolean arrangements $[13]$ ) in $\mathbb{R}^{d}$;

- the permutohedron is the zonotope corresponding to the braid arrangement in $\mathbb{R}^{d}$.

A regular cell complex $\Delta$ is a (combinatorial) zonotopal complex if each cell of $\Delta$ is combinatorially equivalent to a zonotope [13. Analogously, $\Delta$ is a cube complex if each of its cells is a combinatorial cube. A geometric zonotopal or cube complex is a zonotopal (respectively, cube) complex $\Delta$ with a metric such that each face is isometric to a zonotope (respectively, a cube) of the Euclidean space. Moreover, faces are glued together by isometry along their maximal common subfaces. The cell complex $\Delta(\mathcal{L})$ associated to a lopsided set $(E, \mathcal{L})$ is a geometric cube complex: $\Delta(\mathcal{L})$ is the union of all subcubes of the cube $[-1,+1]^{E}$ whose barycenters are sign vectors from $\mathcal{L}[4]$.

A $\operatorname{COM}(E, \mathcal{L})$ is called locally realizable (or zonotopal) if $\mathcal{L}(X)$ is a realizable oriented matroid for any $X \in \mathcal{L}$. Then $\Delta(\mathcal{L})$ is a zonotopal complex because each cell $\Delta(\mathcal{L}(X)), X \in \mathcal{L}$, is combinatorially equivalent to a zonotope. A zonotopal $\operatorname{COM}(E, \mathcal{L})$ is called zonotopally realizable if $\Delta(\mathcal{L})$ is a geometric zonotopal complex. Clearly, zonotopally realizable COMs are locally realizable. The converse is the content of the following question:

Question 1. Is any locally realizable COM zonotopally realizable?

Proposition 16. If $\mathcal{L}$ is a realizable $C O M$, then $\mathcal{L}$ is zonotopally realizable (and thus locally realizable). In particular, each ranking COM is zonotopally realizable.

Proof. Since $\mathcal{L}$ is realizable there is a set of oriented affine hyperplanes of $\mathbb{R}^{d}$ and an open convex set $C$, such that $\mathcal{L}=\mathcal{L}(E, C)$. Without loss of generality we can assume that $C$ is the interior of a full-dimensional polyhedron $P$. Let $F$ be the set of supporting hyperplanes of $P$. Consider the central hyperplane arrangement $A$ resulting from lifting the affine arrangement $E \cup F$ to $\mathbb{R}^{d+1}$. The associated $\mathrm{OM} \mathcal{L}^{\prime}$ is realizable and therefore zonotopally realizable. Since $\Delta(\mathcal{L})$ is a subcomplex of $\Delta\left(\mathcal{L}^{\prime}\right)$, also $\mathcal{L}$ is zonotopally realizable.

11.5. CAT(0) Coxeter COMs. We conclude this section by presenting another class of zonotopally realizable COMs. Namely, we prove that the CAT(0) Coxeter (zonotopal) complexes introduced in 24] arise from COMs. They represent a common generalization of 
benzenoid systems (used for illustration in Section 6), 2-dimensional cell complexes obtained from bipartite cellular graphs [1, and CAT(0) cube complexes (cube complexes arising from median structures) [2]. One can say that CAT(0) zonotopal complexes generalize CAT(0) cube complexes in the same way as COMs generalize lopsided sets.

A zonotope $\mathcal{Z}$ is called a Coxeter zonotope (an even polyhedron [24] or a Coxeter cell [14]) if $\mathcal{Z}$ is symmetric with respect to the mid-hyperplane $H_{f}$ of each edge $f$ of $\mathcal{Z}$, i.e., to the hyperplane perpendicular to $f$ and passing via the middle of $f$. A cell complex $\Delta$ is called a Coxeter complex if $\Delta$ is a geometric zonotopal complex in which each cell is isometric to a Coxeter zonotope. Throughout this subsection, by $\Delta$ we denote a Coxeter complex and by $\|\Delta\|$ the underlying metric space of $\Delta$.

If $\mathcal{Z}$ is a Coxeter zonotope and $f, f^{\prime}$ are two parallel edges of $\mathcal{Z}$, then one can easily see that the mid-hyperplanes $H_{f}$ and $H_{f^{\prime}}$ coincide. If $\mathcal{Z}=\left[-\mathbf{x}_{1}, \mathbf{x}_{1}\right]+\left[-\mathbf{x}_{2}, \mathbf{x}_{2}\right]+\ldots+\left[-\mathbf{x}_{n}, \mathbf{x}_{n}\right]$, denote by $H_{i}$ the mid-hyperplane to all edges of $\mathcal{Z}$ parallel to the segment $\left[-\mathbf{x}_{i}, \mathbf{x}_{i}\right], i=1, \ldots, n$. Then $\mathcal{Z}$ is the zonotope of the regions defined by the arrangement $\left\{H_{1}, \ldots, H_{n}\right\}$. It is wellknown [14, Definition 7.3.1] (and is also noticed in [24, p.184]) that Coxeter zonotopes are exactly the zonotopes associated to reflection arrangements (called also Coxeter arrangements) of hyperplanes, i.e., to arrangements of hyperplanes of a finite reflection group [10, Subsection 2.3]. For each $i=1, \ldots, n$, denote by $\mathcal{Z}_{i}$ the intersection of $\mathcal{Z}$ with the hyperplane $H_{i}$ and call it a mid-section of $\mathcal{Z}$. The mid-sections $\mathcal{Z}_{i}$ of a Coxeter zonotope $\mathcal{Z}$ of dimension $d$ are Coxeter zonotopes of dimension $d-1$.

We continue with the definition of $\operatorname{CAT}(0)$ metric spaces and $\operatorname{CAT}(0)$ Coxeter complexes. The underlying space (polyhedron) $\|\Delta\|$ of a geometric zonotopal complex (and, more generally, of a cell complex with Euclidean convex polytopes as cells) $\Delta$ can be endowed with an intrinsic $l_{2}$-metric in the following way. Assume that inside every maximal face of $\|\Delta\|$ the distance is measured by the $l_{2}$-metric. The intrinsic $l_{2}$-metric $d_{2}$ of $\|\Delta\|$ is defined by letting the distance between two points $x, y \in\|\Delta\|$ be equal to the greatest lower bound on the length of the paths joining them; here a path in $\|\Delta\|$ from $x$ to $y$ is a sequence $x=x_{0}, x_{1}, \ldots, x_{m}=y$ of points in $\|\Delta\|$ such that for each $i=0, \ldots, m-1$ there exists a face $\sigma_{i}$ containing $x_{i}$ and $x_{i+1}$, and the length of the path equals $\sum_{i=0}^{m-1} d\left(x_{i}, x_{i+1}\right)$, where $d\left(x_{i}, x_{i+1}\right)$ is computed inside $\sigma_{i}$ according to the respective $l_{2}$-metric. The resulting metric space is geodesic, i.e., every pair of points in $\|\Delta\|$ can be joined by a geodesic; see [12].

A geodesic triangle $T:=T\left(x_{1}, x_{2}, x_{3}\right)$ in a geodesic metric space $(X, d)$ consists of three points in $X$ (the vertices of $T$ ) and a geodesic between each pair of vertices (the edges of $T)$. A comparison triangle for $T\left(x_{1}, x_{2}, x_{3}\right)$ is a triangle $T\left(x_{1}^{\prime}, x_{2}^{\prime}, x_{3}^{\prime}\right)$ in the Euclidean plane $\mathbb{R}^{2}$ such that $d_{\mathbb{R}^{2}}\left(x_{i}^{\prime}, x_{j}^{\prime}\right)=d\left(x_{i}, x_{j}\right)$ for $i, j \in\{1,2,3\}$. A geodesic metric space $(X, d)$ is a $C A T(0)$ space 23] if all geodesic triangles $T\left(x_{1}, x_{2}, x_{3}\right)$ of $X$ satisfy the comparison axiom of Cartan-Alexandrov-Toponogov: If $y$ is a point on the side of $T\left(x_{1}, x_{2}, x_{3}\right)$ with vertices $x_{1}$ and $x_{2}$ and $y^{\prime}$ is the unique point on the line segment $\left[x_{1}^{\prime}, x_{2}^{\prime}\right]$ of the comparison triangle $T\left(x_{1}^{\prime}, x_{2}^{\prime}, x_{3}^{\prime}\right)$ such that $d_{\mathbb{R}^{2}}\left(x_{i}^{\prime}, y^{\prime}\right)=d\left(x_{i}, y\right)$ for $i=1,2$, then $d\left(x_{3}, y\right) \leq d_{\mathbb{R}^{2}}\left(x_{3}^{\prime}, y^{\prime}\right)$.

$\mathrm{CAT}(0)$ spaces can be characterized in several different natural ways and have numerous properties (for a full account of this theory consult the book 12]). For instance, a cell complex 
endowed with a piecewise Euclidean metric is $\operatorname{CAT}(0)$ if and only if any two points can be joined by a unique geodesic. Moreover, $\mathrm{CAT}(0)$ spaces are contractible.

A CAT(0) Coxeter complex is a Coxeter complex $\Delta$ for which $\|\Delta\|$ endowed with the intrinsic $l_{2}$-metric $d_{2}$ is a CAT(0) space. In this case, the parallelism relation on edges of cells of $\Delta$ induces a parallelism relation on all edges of $\Delta$ : two edges $f, f^{\prime}$ of $\Delta$ are parallel if there exists a sequence of edges $f_{0}=f, f_{1}, \ldots, f_{k-1}, f_{k}=f^{\prime}$ such that any two consecutive edges $f_{i-1}, f_{i}$ are parallel edges of a common cell of $\Delta$. Parallelism is an equivalence relation on the edges of $\Delta$. Denote by $E$ the equivalence classes of this parallelism relation. For $e \in E$, we denote by $\Delta_{e}$ the union of all mid-sections of the form $\mathcal{Z}_{e}$ for cells $\mathcal{Z} \in \Delta$ which contain edges from the equivalence class $e$ (let $\left\|\Delta_{e}\right\|$ be the underlying space of $\Delta_{e}$ ). We call each $\left\|\Delta_{e}\right\|$ (or $\Delta_{e}$ ), $e \in E$, a mid-hyperplane (or a wall as in [24]) of $\|\Delta\|$. Since each mid-section included in $\Delta_{e}$ is a Coxeter zonotope, each mid-hyperplane of a Coxeter complex is a Coxeter complex as well. CAT(0) Coxeter complexes have additional nice and strong properties, which have been established in 24.

Lemma 6. [24, Lemme 4.4] Let $\Delta$ be a CAT(0) Coxeter complex and $\Delta_{e}$ be a mid-hyperplane of $\Delta$. Then $\left\|\Delta_{e}\right\|$ is a convex subset of $\|\Delta\|$ and $\left\|\Delta_{e}\right\|$ partitions $\|\Delta\|$ in two connected components $\left\|\Delta_{e}^{-}\right\|$and $\left\|\Delta_{e}^{+}\right\|$(called halfspaces of $\|\Delta\|$ ).

If $x \in\left\|\Delta_{e}^{-}\right\|$and $y \in\left\|\Delta_{e}^{+}\right\|$, then $x$ and $y$ are said to be separated by the mid-hyperplane (wall) $\left\|\Delta_{e}\right\|$. A path $P$ in $\|\Delta\|$ traverses a mid-hyperplane $\left\|\Delta_{e}\right\|$ if $P$ contains an edge $x y$ such that $x$ and $y$ are separated by $\left\|\Delta_{e}\right\|$. Two distinct mid-hyperplanes $\left\|\Delta_{e}\right\|$ and $\left\|\Delta_{f}\right\|$ are called parallel if $\left\|\Delta_{e}\right\| \cap\left\|\Delta_{f}\right\|=\varnothing$ and crossing if $\left\|\Delta_{e}\right\| \cap\left\|\Delta_{f}\right\| \neq \varnothing$.

Lemma 7. [24, Corollaire 4.10] Two vertices $u, v$ of $\Delta$ are adjacent in $G(\Delta)$ if and only if $u$ and $v$ are separated by a single mid-hyperplane of $\|\Delta\|$.

Lemma 8. [24, Proposition 4.11] A path $P$ of $G(\Delta)$ between two vertices $u, v$ is a shortest $(u, v)$-path in $G(\Delta)$ if and only if $P$ traverses each mid-hyperplane of $\|\Delta\|$ at most once.

These three results imply that the arrangement of mid-hyperplanes of a CAT(0) Coxeter complex $\Delta$ defines a wall system sensu [24], which in turn provides us with a system $\mathcal{L}(\Delta)$ of sign vectors. Define the mapping $\varphi: \Delta \rightarrow\{ \pm 1,0\}^{E}$ in the following way. First, for $e \in E$ and $x \in \Delta$, set

$$
\varphi_{e}(x):= \begin{cases}-1 & \text { if } x \in\left\|\Delta_{e}^{-}\right\|, \\ 0 & \text { if } x \in\left\|\Delta_{e}\right\|, \\ +1 & \text { if } x \in\left\|\Delta_{e}^{+}\right\| .\end{cases}
$$

Let $\varphi(x)=\left(\varphi_{e}(x): e \in E\right)$. Denote by $\mathcal{L}(\Delta)$ the set of all sign vectors of the form $\varphi(x), x \in$ $\|\Delta\|$. Notice that if a point $x$ of $\|\Delta\|$ does not belong to any mid-hyperplane of $\|\Delta\|$, then $\varphi(x) \in\{ \pm 1\}^{E}$; in particular, this is the case for the vertices of $G(\Delta)$. Moreover, Lemma 8 implies that $\varphi$ defines an isometric embedding of $G(\Delta)$ into the hypercube $\{ \pm 1\}^{E}$. 
Theorem 5. Let $\Delta$ be a CAT(0) Coxeter complex, $E$ be the classes of parallel edges of $\Delta$, and $\mathcal{L}(\Delta):=\cup\{\varphi(x): x \in\|\Delta\|\} \subseteq\{ \pm 1,0\}^{E}$. Then $(E, \mathcal{L}(\Delta))$ is a simple COM and $G(\Delta)$ is its tope graph.

Proof. We proceed by induction on the size of $E$. It suffices to show that $(E, \mathcal{L}(\Delta))$ is simple and satisfies the conditions $(1),(3),(4)$, and $\left(2^{\prime}\right)$ of Theorem 3 . That $\mathcal{L}(\Delta)$ satisfies (N1) is evident. To verify the condition (N2), let $e, f \in E$. If the mid-hyperplanes $\left\|\Delta_{e}\right\|$ and $\left\|\Delta_{f}\right\|$ cross, then the four intersections $\left\|\Delta_{e}^{-}\right\| \cap\left\|\Delta_{f}^{-}\right\|,\left\|\Delta_{e}^{-}\right\| \cap\left\|\Delta_{e}^{+}\right\|,\left\|\Delta_{e}^{+}\right\| \cap\left\|\Delta_{f}^{-}\right\|$, and $\left\|\Delta_{e}^{+}\right\| \cap\left\|\Delta_{f}^{+}\right\|$are nonempty, and as $X$ and $Y$ with $\left\{X_{e} X_{f}, Y_{e} Y_{f}\right\}=\{ \pm 1\}$ one can pick the sign vectors $\varphi(x)$ and $\varphi(y)$ of any two points $x \in\left\|\Delta_{e}^{+}\right\| \cap\left\|\Delta_{f}^{-}\right\|$and $y \in\left\|\Delta_{e}^{-}\right\| \cap\left\|\Delta_{f}^{-}\right\|$. Otherwise, if $\left\|\Delta_{e}\right\|$ and $\left\|\Delta_{f}\right\|$ are parallel, then one of the four pairwise intersections of halfspaces is empty, say $\left\|\Delta_{e}^{-}\right\| \cap\left\|\Delta_{f}^{+}\right\|=\varnothing$, and as $X$ and $Y$ one can take the sign vectors $\varphi(x)$ and $\varphi(y)$ of any points $x \in\left\|\Delta_{e}^{+}\right\| \cap\left\|\Delta_{f}^{+}\right\|$and $y \in\left\|\Delta_{e}^{+}\right\| \cap\left\|\Delta_{f}^{-}\right\|$. This establishes that $(E, \mathcal{L}(\Delta))$ is simple.

Notice that the tope graph of $(E, \mathcal{L}(\Delta))$ coincides with $G(\Delta)$. Indeed, let $X$ be a tope of $\mathcal{L}(\Delta)$. Then $X=\varphi(x)$ for some $x \in\|\Delta\|$. Let $x \in \mathcal{Z}$ for a cell $\mathcal{Z}$ of $\|\Delta\|$. The sign maps of $\mathcal{L}(\Delta)$ restricted to $\mathcal{Z}$ define an oriented matroid whose topes are the vertices of $\mathcal{Z}$. Therefore $\mathcal{Z}$ contains a vertex $v$ such that $\varphi(v)=\varphi(x)=X$, whence each tope of $\mathcal{L}(\Delta)$ is a vertex of $G(\Delta)$. Conversely, since each vertex $v$ of $G(\Delta)$ does not belong to any mid-hyperplane of $\|\Delta\|, \varphi(v)$ is a vertex of $\{ \pm 1\}^{E}$, and thus a tope of $\mathcal{L}(\Delta)$. This shows that the tope graph of $\mathcal{L}(\Delta)$ and the 1-skeleton of $\Delta$ have the same sets of vertices. Lemma 7 implies that two vertices $u$ and $v$ are adjacent in $G(\Delta)$ if and only if they are adjacent in the tope graph of $\mathcal{L}(\Delta)$. Then Lemma 8 establishes the condition (3). The condition (4) immediately follows from the definition of $\mathcal{L}(\Delta)$.

Now we establish condition $\left(2^{\prime}\right)$ that all hyperplanes $\mathcal{L}_{e}^{0}(\Delta)$ of $(E, \mathcal{L}(\Delta))$ are COMs. Notice that $X \in \mathcal{L}_{e}^{0}(\Delta)$ if and only if $X=\varphi(x)$ for some $x \in\left\|\Delta_{e}\right\|$. Therefore the hyperplane $\mathcal{L}_{e}^{0}(\Delta)$ of $\mathcal{L}(\Delta)$ coincides with the restriction of $\mathcal{L}(\Delta)$ to the points of the mid-hyperplane $\left\|\Delta_{e}\right\|$. Hence $\mathcal{L}_{e}^{0}(\Delta)$ can be viewed as $\mathcal{L}\left(\Delta_{e}\right)$, where $\mathcal{L}\left(\Delta_{e}\right)$ is the set of all sign vectors of $\{ \pm 1,0\}^{E}$ of the form $\varphi(x), x \in\left\|\Delta_{e}\right\|$. By Lemma 6 , $\left\|\Delta_{e}\right\|$ is a convex subset of $\|\Delta\|$, thus $\Delta_{e}$ is a CAT(0) Coxeter complex. Let $E^{\prime}$ denote the classes of parallel edges of $\Delta_{e}$; namely, $E^{\prime}$ consists of all $f \in E \backslash\{e\}$ such that the mid-hyperplanes $\left\|\Delta_{e}\right\|$ and $\left\|\Delta_{f}\right\|$ are crossing. Notice that the $f$ th mid-hyperplane $\left\|\left(\Delta_{e}\right)_{f}\right\|$ of $\left\|\Delta_{e}\right\|$ is just the intersection $\left\|\Delta_{e}\right\| \cap\left\|\Delta_{f}\right\|$. Analogously, the halfspaces $\left\|\left(\Delta_{e}\right)_{f}^{-}\right\|$and $\left\|\left(\Delta_{e}\right)_{f}^{+}\right\|$coincide with the intersections $\left\|\Delta_{f}^{-}\right\| \cap\left\|\Delta_{e}\right\|$ and $\left\|\Delta_{f}^{+}\right\| \cap$ $\left\|\Delta_{e}\right\|$, respectively. Define $\varphi^{\prime}:\left\|\Delta_{e}\right\| \rightarrow\{ \pm 1,0\}^{E^{\prime}}$ as follows. For $x \in\left\|\Delta_{e}\right\|$ and $f \in E^{\prime}$, set

$$
\varphi_{f}^{\prime}(x):= \begin{cases}-1 & \text { if } x \in\left\|\left(\Delta_{e}\right)_{f}^{-}\right\|, \\ 0 & \text { if } x \in\left\|\left(\Delta_{e}\right)_{f}\right\|, \\ +1 & \text { if } x \in\left\|\left(\Delta_{e}\right)_{f}^{+}\right\| .\end{cases}
$$

Let $\varphi^{\prime}(x)=\left(\varphi_{f}^{\prime}(x): f \in E^{\prime}\right)$. Denote by $\mathcal{L}^{\prime}\left(\Delta_{e}\right)$ the set of all sign vectors of the form $\varphi^{\prime}(x), x \in\left\|\Delta_{e}\right\|$. By the induction hypothesis, $\left(E^{\prime}, \mathcal{L}^{\prime}\left(\Delta_{e}\right)\right)$ is a COM. For any point $x \in$ $\left\|\Delta_{e}\right\|, \varphi(x)$ coincides with $\varphi^{\prime}(x)$ on $E^{\prime}$. Since $\varphi_{e}(x)=0, \varphi_{e^{\prime}}(x)=-1$ if $\left\|\Delta_{e^{\prime}}\right\|$ is parallel to 
$\left\|\Delta_{e}\right\|$ and $\left\|\Delta_{e}\right\| \subset\left\|\Delta_{e^{\prime}}^{-}\right\|$, and $\varphi_{e^{\prime}}(x)=+1$ if $\left\|\Delta_{e^{\prime}}\right\|$ is parallel to $\left\|\Delta_{e}\right\|$ and $\left\|\Delta_{e}\right\| \subset\left\|\Delta_{e^{\prime}}^{+}\right\|$, $\mathcal{L}\left(\Delta_{e}\right)$ can be obtained from $\mathcal{L}^{\prime}\left(\Delta_{e}\right)$ by adding to all sign vectors of $\mathcal{L}^{\prime}\left(\Delta_{e}\right)$ in each coordinate of $E \backslash E^{\prime}$ a respective constant $0,-1$, or +1 . Hence $\left(E, \mathcal{L}\left(\Delta_{e}\right)\right)$ is a COM, thus establishing $\left(2^{\prime}\right)$.

Finally, we show that $\mathcal{L}(\Delta)$ satisfies the condition (1), i.e., the composition rule (C). Let $X$ and $Y$ be two sign vectors of $\mathcal{L}(\Delta)$ and $x$ and $y$ be two points of $\|\Delta\|$ such that $\varphi(x)=X$ and $\varphi(y)=Y$. As in the case of realizable COMs presented in the introduction, connect the two points $x$ and $y$ by the unique geodesic $\gamma(x, y)$ of $\|\Delta\|$ and choose $\epsilon>0$ small enough so that the open ball of radius $\epsilon$ around $x$ intersects only those mid-hyperplanes of $\|\Delta\|$ on which $x$ lies. Pick any point $w$ from the intersection of this $\epsilon$-ball with $\gamma(x, y) \backslash\{x\}$ and let $W=\varphi(w)$. We assert that $W=X \circ Y$. Pick any $e \in E$. First suppose that $X_{e} \neq 0$. From the choice of $w$ it immediately follows that $W_{e}=\varphi_{e}(w)=\varphi_{e}(x)=X_{e}$. Now suppose that $X_{e}=0$. If $Y_{e}=0$, then $x, y \in\left\|\Delta_{e}\right\|$. Since by Lemma 6, $\left\|\Delta_{e}\right\|$ is a convex subset of $\|\Delta\|$, we have $w \in \gamma(x, y) \subset\left\|\Delta_{e}\right\|$, whence $W_{e}=0=X_{e} \circ Y_{e}$. Finally, if $Y_{e} \neq 0$, say $Y_{e}=+1$, then since the set $\left\|\Delta_{e}^{+}\right\| \cup\left\|\Delta_{e}\right\|$ is convex, either $w \in\left\|\Delta_{e}^{+}\right\|$or $w \in\left\|\Delta_{e}\right\|$. In the first case, we have $W_{e}=+1=X_{e} \circ Y_{e}$ and we are done. On the other hand, we will show below that the case $w \in\left\|\Delta_{e}\right\|$ is impossible.

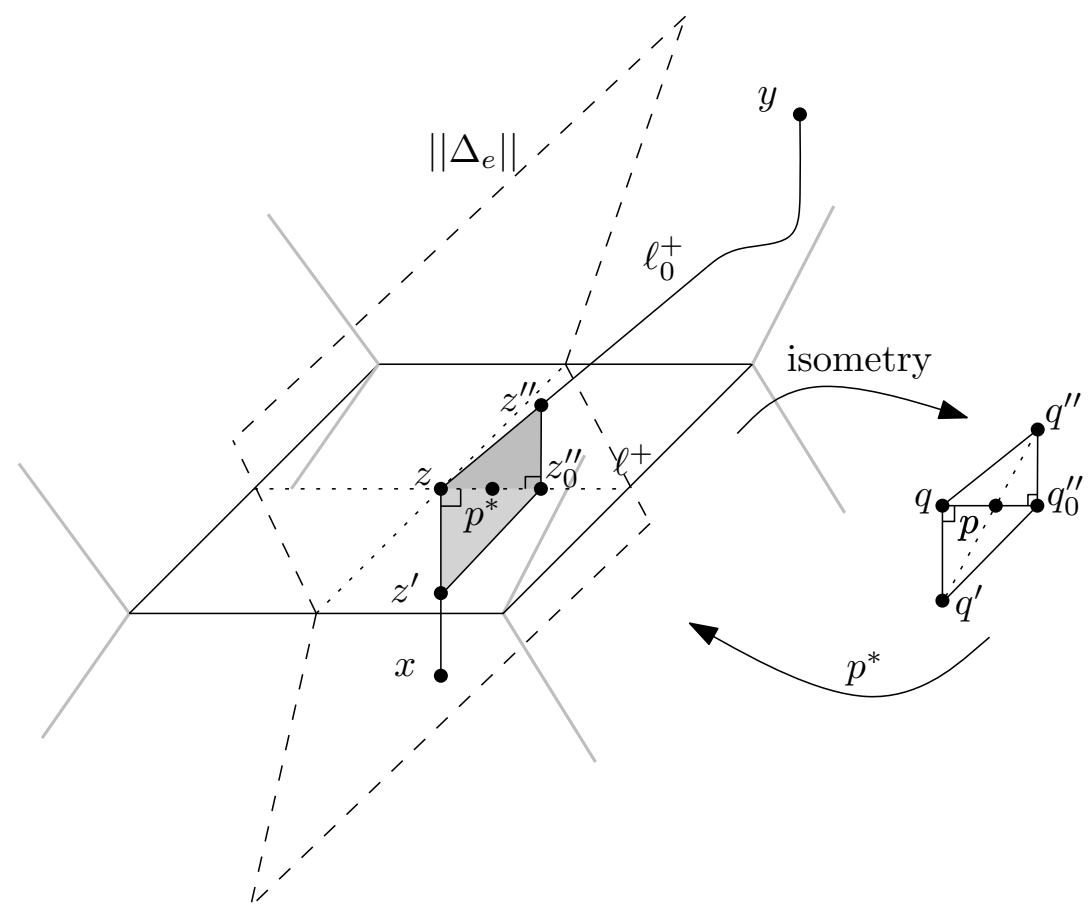

Figure 6. To the proof of composition rule in Theorem 5 .

Indeed, suppose by way of contradiction that $w \in\left\|\Delta_{e}\right\|$. Since $x \in\left\|\Delta_{e}\right\|, y \in\left\|\Delta_{e}^{+}\right\|$, and $\left\|\Delta_{e}\right\|$ is convex, there exists a point $z \in \gamma(x, y)$ such that $\gamma(x, z) \subset\left\|\Delta_{e}\right\|$ and $\gamma(z, y) \backslash\{z\} \subset$ 
$\left\|\Delta_{e}^{+}\right\|$. Pick points $z^{\prime} \in \gamma(x, z)$ and $z^{\prime \prime} \in \gamma(z, y)$ close enough to $z$ such that each of the couples $z^{\prime}, z$ and $z, z^{\prime \prime}$ belongs to a common cell of $\|\Delta\|$. The choice of $z$ implies that $z^{\prime}, z, z^{\prime \prime}$ cannot all belong to a common cell of $\|\Delta\|$. Denote by $\mathcal{Z}^{\prime}$ a maximal cell containing $z^{\prime}, z$ and by $\mathcal{Z}^{\prime \prime}$ a maximal cell containing $z, z^{\prime \prime}$. Then $z$ belongs to a common face $\mathcal{Z}_{0}$ of $\mathcal{Z}^{\prime}$ and $\mathcal{Z}^{\prime \prime}$. Let $\Pi^{\prime}$ and $\Pi^{\prime \prime}$ denote the supporting Euclidean spaces of $\mathcal{Z}^{\prime}$ and $\mathcal{Z}^{\prime \prime}$, respectively. Let $\ell$ be the line in $\Pi^{\prime \prime}$ passing via the point $z$ and parallel to the edges of $\mathcal{Z}^{\prime \prime}$ from the equivalence class $e$ and let $\ell^{+}$be the ray with origin $z$ and containing the points of $\ell \cap\left\|\Delta_{e}^{+}\right\|$(this intersection is a non-empty half-open interval). Let $\ell_{0}$ be the line in $\Pi^{\prime \prime}$ passing via $z$ and $z^{\prime \prime}$ and let $\ell_{0}^{+}$ be the ray of $\ell_{0}$ with origin $z$ and containing $\left[z, z^{\prime \prime}\right]$. Since $\ell$ is orthogonal to the supporting plane of the mid-section $\mathcal{Z}_{0}^{\prime}=\mathcal{Z}^{\prime} \cap \Delta_{e}$, the angle between $\ell_{0}^{+}$and $\left[z, z^{\prime}\right]$ is $\frac{\pi}{2}$. Now suppose that $z^{\prime \prime}$ is selected so close to $z$ that the orthogonal projection $z_{0}^{\prime \prime}$ of $z^{\prime \prime}$ on the line $\ell$ belongs to the intersection $\mathcal{Z}_{0} \cap \ell^{+}$.

As a result, we obtain two right-angled triangles $T\left(z, z^{\prime}, z_{0}^{\prime \prime}\right)$ and $T\left(z, z^{\prime \prime}, z_{0}^{\prime \prime}\right)$, the first belonging to $\Pi^{\prime}$ and the second belonging to $\Pi^{\prime \prime}$ (see Figure 6 for an illustration). Therefore, their union is isometric to a convex quadrilateral $Q=Q\left(q, q^{\prime}, q_{0}^{\prime \prime}, q^{\prime \prime}\right)$ in $\mathbb{R}^{2}$ having the sides $q q^{\prime}, q^{\prime} q_{0}^{\prime \prime}, q_{0}^{\prime \prime} q^{\prime \prime}$, and $q^{\prime \prime} q$ of lengths $d_{2}\left(z, z^{\prime}\right), d_{2}\left(z^{\prime}, z_{0}^{\prime \prime}\right), d_{2}\left(z_{0}^{\prime \prime}, z^{\prime \prime}\right)$, and $d_{2}\left(z^{\prime \prime}, z\right)$, respectively. Let $p$ be the intersection of the diagonals $q^{\prime} q^{\prime \prime}$ and $q q_{0}^{\prime \prime}$ of $Q$ and let $p^{*}$ be the point (of $\Delta$ ) on the segment $\left[z, z_{0}^{\prime \prime}\right]$ such that $d_{2}\left(z, p^{*}\right)=d_{\mathbb{R}^{2}}(q, p)$ and $d_{2}\left(z_{0}^{\prime \prime}, p^{*}\right)=d_{\mathbb{R}^{2}}\left(q_{0}^{\prime \prime}, p\right)$. Then

$$
\begin{aligned}
& d_{2}\left(z^{\prime}, z\right)+d_{2}\left(z, z^{\prime \prime}\right)=d_{\mathbb{R}^{2}}\left(q^{\prime}, q\right)+d_{\mathbb{R}^{2}}\left(q, q^{\prime \prime}\right)>d_{\mathbb{R}^{2}}\left(q^{\prime}, q^{\prime \prime}\right) \\
& =d_{\mathbb{R}^{2}}\left(q^{\prime}, p\right)+d_{\mathbb{R}^{2}}\left(p, q^{\prime \prime}\right)=d_{2}\left(z^{\prime}, p^{*}\right)+d_{2}\left(p^{*}, z^{\prime \prime}\right),
\end{aligned}
$$

contrary to the assumption that $z \in \gamma\left(z^{\prime}, z^{\prime \prime}\right) \subset \gamma(x, y)$. This establishes our claim and concludes the proof that $\mathcal{L}(\Delta)$ satisfies the composition rule $(\mathrm{C})$.

We conclude this section by showing that in fact all COMs having square-free tope graphs arise from 2-dimensional zonotopal COMs:

Proposition 17. A square-free partial cube $G$ is the tope graph of a $C O M \mathcal{L}(G)$ if and only if $G$ does not contain an 8-cycle with two subdivided diagonal chords (graph $X$ in Fig.1 in [30]) as an isometric subgraph. The resulting $\mathcal{L}(G)$ is a zonotopal COM.

Proof. Notice that a square-free tope graph $G$ of a COM does not contain $X$ as an isometric subgraph. Indeed, since $G$ is square-free, the four 6-cycles of $X$ are convex and, moreover, $X$ must be a convex subgraph of $G$. Since $X$ isometrically embeds into a 4 -cube, it can be directly checked that $X$ is not the tope graph as a COM, consequently, $X$ cannot occur in the tope graph of a COM.

Conversely, let $G$ be a square-free partial cube not containing $X$ as an isometric subgraph. By Proposition 2.6. of [30], any pair of isometric cycles intersect in at most one edge. By replacing each isometric cycle of $G$ with a regular polygon with the same number of edges, we get a 2-dimensional Coxeter zonotopal complex $\|\Delta(G)\|$. Since isometric cycles of a graph generate the cycle space, this complex is simply connected. Since the sum of angles around any vertex of $\|\Delta(G)\|$ is at least $2 \pi$, by Gromov's result for 2-dimensional complexes [12, p.215], 
$\|\Delta(G)\|$ is $\operatorname{CAT}(0)$. Thus, $\Delta(G)$ is a 2-dimensional CAT(0) Coxeter zonotopal complex and by Theorem 5 , $G$ can be realized as the tope graph of a COM.

\section{Concluding Remarks}

In this paper, we show how COMs naturally arise as a generalization of oriented matroids and lopsided sets by relaxing the covector axioms. Furthermore, we give several descriptions of COMs, in particular, in terms of cocircuit axioms. Nevertheless, such important features of the theory of OMs like duality and topological representation still lack generalization. We believe that the following problem, which is well-known for OMs and lopsided sets, is an important next step.

Problem 1. Establish duality theory for COMs.

By Proposition 6, the halfspaces of a COM are COMs. Particular examples are the affine oriented matroids, which are halfspaces of OMs. Even stronger, Lemma 4 shows that the intersections of halfspaces, i.e., the fibers, of a COM are COMs. While the following is true by definition for realizable COMs (see for instance the proof of Proposition 16), we believe that every $\mathrm{COM}$ arises this way from an OM:

Conjecture 1. Every COM is a fiber of some OM.

This generalizes the corresponding conjecture of Lawrence 29 that lopsided sets are fibers of uniform OMs. Not only would Conjecture 1 be a natural generalization of the realizable situation, but using the Topological Representation Theorem of Oriented Matroids [21] it will also give a natural topological representation for COMs. In fact, Conjecture 1 is equivalent to the following conjecture: For every $\operatorname{COM} \mathcal{L}$ there is a number $d$ such that $\mathcal{L}$ can be represented by a set of $(d-2)$-dimensional pseudospheres restricted to the intersection of a set of open $(d-1)$-dimensional pseudoballs inside a $(d-1)$-sphere.

For locally realizable COMs, the following version of Conjecture 1 would imply a positive answer to Question 1 .

Conjecture 2. Every locally realizable COM is a fiber of a realizable OM.

Conjecture 2 can be rephrased as: The tope graph of a locally realizable COM is a convex subgraph of the 1-skeleton of a zonotope. Analogously, Conjecture 11 can be rephrased as: The tope graph of a COM is a convex subgraph of the tope graph of an OM.

Acknowledgements. We would like to acknowledge the referees for useful remarks improving the presentation. Furthermore, we thank Matjaž Kovše (Leipzig) for initial discussions about partial cubes and tope graphs of oriented matroids and are grateful to Andrea Baum, Tabea Beese, and especially Yida Zhu (Hamburg) for careful reading of previous versions and suggesting a simpler argument in the proof of Theorem 1. V.C. and K.K. were partially supported by ANR project TEOMATRO (ANR-10-BLAN-0207). K.K was furthermore supported by PEPS grant EROS and ANR project GATO (ANR-16-CE40-0009-01). 


\section{REFERENCES}

[1] H.-J. Bandelt and V. Chepoi, Cellular bipartite graphs, Europ. J. Combin., 17 (1996), pp. 121-134.

[2] H.-J. BAndelt And V. ChePoi, Metric graph theory and geometry: a survey, in Surveys on discrete and computational geometry, vol. 453 of Contemp. Math., Amer. Math. Soc., Providence, RI, 2008, pp. 49-86.

[3] H.-J. Bandelt, V. Chepoi, A. Dress, and J. Koolen, Combinatorics of lopsided sets, Europ. J. Combin., 27 (2006), pp. 669-689.

[4] H.-J. Bandelt, V. Chepoi, A. Dress, and J. Koolen, Geometry of lopsided sets, 2013. Preprint.

[5] A. BAUm AND Y. ZHU, The axiomatization of affine oriented matroids reassessed, arXiv, (2016).

[6] R. Bignall, A non-commutative multiple-valued logic, in Proceedings of the Twenty-First International Symposium on Multiple-Valued Logic, D. Miller, ed., IEEE Computer Society Press, Los Alamitos, 1991, pp. 49-54.

[7] A. BJÖRner, Topological methods, in Handbook of combinatorics, Vol. 1, 2, Elsevier, 1995, pp. $1819-1872$.

[8] A. BJÖRNER, Random walks, arrangements, cell complexes, greedoids, and self-organizing libraries, in Building bridges, Springer, 2008, pp. 165-203.

[9] A. Björner, P. H. Edelman, And G. M. Ziegler, Hyperplane arrangements with a lattice of regions., Discr. Comput. Geom., 5 (1990), pp. 263-288.

[10] A. Björner, M. Las Vergnas, B. Sturmfels, N. White, and G. M. Ziegler, Oriented matroids, vol. 46 of Encyclopedia of Mathematics and its Applications, Cambridge University Press, Cambridge, second ed., 1999.

[11] R. Bland and M. Las Vergnas, Orientability of matroids, J. Combin. Th. Ser. B, 23 (1978), pp. $94-123$.

[12] M. R. BRidson And A. HAefliger, Metric spaces of non-positive curvature, vol. 319 of Grundlehren der Mathematischen Wissenschaften, Springer-Verlag, Berlin, 1999.

[13] M. Davis, T. Januszkiewics, And R. Scott, Nonpositive curvature and blow-ups, Selecta Mathematica, 11 (1998), pp. 491-547.

[14] M. W. DAVIS, The geometry and topology of Coxeter groups, vol. 32 of London Mathematical Society Monographs Series, Princeton University Press, 2008.

[15] M. Deza And K. Fukuda, On bouquets of matroids and orientations, Kokyuroku, Research Institute for Mathematical Sciences, Kyoto University, 587 (1986), pp. 110-129.

[16] M. Deza And M. Laurent, Geometry of cuts and metrics, vol. 15 of Algorithms and Combinatorics, Springer-Verlag, 1997.

[17] R. P. Dilworth, A decomposition theorem for partially ordered sets, Ann. of Math. (2), 51 (1950), pp. $161-166$.

[18] X. Dong, On the bounded complex of an affine oriented matroid, Discr. Comput. Geom., 35 (2006), pp. $457-471$.

[19] X. Dong, The bounded complex of a uniform affine oriented matroid is a ball, J. Combin. Th. Ser. A, 115 (2008), pp. 651-661.

[20] J. Edmonds And A. MANDEL, Topology of oriented matroids, (1982).

[21] J. Folkman and J. Lawrence, Oriented matroids, J. Combin. Th. Ser. B, 25 (1978), pp. $199-236$.

[22] L. Fuchs, Partially ordered algebraic systems, Pergamon Press, Oxford-London-New York-Paris; Addison-Wesley Publishing Co., Inc., Reading, Mass.-Palo Alto, Calif.-London, 1963.

[23] M. Gromov, Hyperbolic groups, in Essays in group theory, vol. 8 of Math. Sci. Res. Inst. Publ., Springer, 1987, pp. $75-263$.

[24] F. Haglund and F. Paulin, Simplicité de groupes d'automorphismes d'espaces à courbure négative, in The Epstein birthday schrift, vol. 1 of Geom. Topol. Monogr., Geom. Topol. Publ., Coventry, 1998, pp. 181-248 (electronic).

[25] R. Hammack, W. Imrich, And S. KlavžAR, Handbook of product graphs, Discrete Mathematics and its Applications (Boca Raton), CRC Press, Boca Raton, FL, 2011. 
[26] K. Handa, Topes of oriented matroids and related structures, Publ. Res. Inst. Math. Sci., 29 (1993), pp. 235-266.

[27] J. KARLANDER, A characterization of affine sign vector systems, in $\mathrm{PhD}$ Thesis, Kungliga Tekniska Högskolan Stockholm, 1992, pp. 67-91.

[28] M. Las Vergnas, Convexity in oriented matroids., J. Comb. Th. Ser. B, 29 (1980), pp. 231-243.

[29] J. LAWREncE, Lopsided sets and orthant-intersection by convex sets, Pacific J. Math., 104 (1983), pp. 155173.

[30] T. Marc, There are no finite partial cubes of girth more than 6 and minimum degree at least 3, Europ. J. Combin., 55 (2016), pp. 62-72.

[31] S. Margolis, F. Saliola, And B. Steinberg, Semigroups embeddable in hyperplane face monoids., Semigroup Forum, 89 (2014), pp. 236-248.

[32] S. Margolis, F. Saliola, And B. Steinberg, Cell complexes, poset topology and the representation theory of algebras arising in algebraic combinatorics and discrete geometry, ArXiv e-prints, (2015).

[33] M. MAssow, Linear extension graphs and linear extension diameter, Dissertation, Technische Universität Berlin, 2009.

[34] S. Moran, Shattering-extremal systems, Mater Thesis, Saarland University, 2012.

[35] M. NAATz, The graph of linear extensions revisited, SIAM J. Discr. Math., 13 (2000), pp. 354-369.

[36] K. Reuter, Linear extensions of a poset as abstract convex sets, Hamburger Beiträge zur Mathematik, 56 (1996).

[37] R. P. Stanley, Two poset polytopes, Discr. Comput. Geom., 1 (1986), pp. 9-23.

[38] D. Wiedeman, Hamming geometry, PhD Thesis, University of Ontario, 1986. re-typeset (http:// plumbyte.com.doug.wiedemann.thesis.pdf), July, 2006.

[39] G. Ziegler, Lectures on polytopes, Graduate Texts in Mathematics, 152, Springer, New York, 7th printing ed., 2007. 